\title{
An Army Marches on Its Stomach: Metabolic Intermediates as Antimicrobial Mediators in Mycobacterium tuberculosis Infection
}

\section{OPEN ACCESS}

Edited by:

Maria Laura Gennaro, Rutgers University, Newark,

United States

Reviewed by:

Selvakumar Subbian Public Health Research Institute

(PHRI), United States Jordi B. Torrelles,

Texas Biomedical Research Institute United States

*Correspondence:

Frederick J. Sheedy fsheedy@tcd.ie

Specialty section:

This article was submitted to

Bacteria and Host.

a section of the journal

Frontiers in Cellular and Infection Microbiology

Received: 28 May 2020

Accepted: 21 July 2020

Published: 25 August 2020

Citation:

Hackett EE and Sheedy FJ (2020) An

Army Marches on Its Stomach: Metabolic Intermediates as

Antimicrobial Mediators in

Mycobacterium tuberculosis Infection.

Front. Cell. Infect. Microbiol. 10:446. doi: 10.3389/fcimb.2020.00446

\author{
Emer E. Hackett and Frederick J. Sheedy* \\ Macrophage Homeostasis, School of Biochemistry and Immunology, Trinity Biomedical Sciences Institute, Trinity College \\ Dublin, Dublin, Ireland
}

The cells of the immune system are reliant on their metabolic state to launch effective responses to combat mycobacterial infections. The bioenergetic profile of the cell determines the molecular fuels and metabolites available to the host, as well as to the bacterial invader. How cells utilize the nutrients in their microenvironment-including glucose, lipids and amino acids - to sustain their functions and produce antimicrobial metabolites, and how mycobacteria exploit this to evade the immune system is of great interest. Changes in flux through metabolic pathways alters the intermediate metabolites present. These intermediates are beginning to be recognized as key modulators of immune signaling as well as direct antimicrobial effectors, and their impact on tuberculosis infection is becoming apparent. A better understanding of how metabolism impacts immunity to Mycobacterium tuberculosis and how it is regulated and thus can be manipulated will open the potential for novel therapeutic interventions and vaccination strategies.

Keywords: tuberculosis, metabolism, macrophage, immunometabolism, immunity, glycolysis, Krebs, antimicrobial

\section{INTRODUCTION}

There is an urgent need for novel interventions for the prevention and treatment of Mycobacterium tuberculosis (Mtb). The current epidemic of Mtb carries a huge cost globally. The World Health Organization's (WHO) 2019 report highlighted the fact that Mtb is the world's most lethal pathogen, responsible for 1.5 million deaths in 2018 (World Health Organization, 2019). It also represents a huge financial burden to those who fall ill in low- and middle-income countries, equating to a $50 \%$ loss of the household income, thus impeding progress in these emergent nations (Tanimura et al., 2014). Of great concern is the increasing incidence of drug resistant Mtb, with over half a million new cases reported in 2018. With these most current statistics, the global community does not look set to reach the WHO End TB Strategy's 2020 milestone of a 20\% decrease in Mtb incidence, achieving only a $6.3 \%$ between 2015 and 2018. The shortcoming on reaching this target is very likely to be worsened by the disruption to healthcare access caused by the COVID-19 outbreak (Manyazewal et al., 2020).

These figures make the case for a new approach to tackling tuberculosis (TB). Vaccination has thus far failed to provide sufficient protection (Dockrell and Smith, 2017) and current treatments fall short, with long treatment regimes, significant pulmonary damage despite curation, ineffective 
preventative treatments for latent $\mathrm{TB}$ and the ever-increasing prominence of drug-resistance (Sulis et al., 2016). This highlights the need for new host-directed therapies (HDTs) that can improve the immune response to $\mathrm{Mtb}$ infection, resulting in the efficient clearance of infection while minimizing damage to host tissues. However, many proposed immunotherapeutics such as monoclonal antibodies and recombinant cytokines are both labile and expensive, thus may not be well-suited to many of the regions in which TB is endemic such as Sub-Saharan Africa and SouthEast Asia. HDTs which target cellular metabolism, particularly existing small molecule therapeutics which can be repurposed, may have the added advantage of being more cost-effective. To develop new therapeutic approaches, the interactions between the microbe and the host must be better characterized.

\section{MYCOBACTERIUM TUBERCULOSIS, MACROPHAGES, AND METABOLISM \\ Macrophage Responses to Mycobacterium tuberculosis}

When an aerosolized droplet containing viable Mtb bacilli is inhaled, it travels to the lower lung and is phagocytosed by the primary host cell for Mtb, the alveolar macrophage. The macrophage is a pivotal immune cell in Mtb infection, responsible for bacterial killing and the instruction of other immune cells. Macrophages are highly plastic, capable of taking on a range of phenotypes when activated depending on their microenvironment (Biswas et al., 2012). In the context of Mtb infection, a spectrum of macrophage activation states is induced (Skold and Behar, 2008), and this changes over time. It has been shown that macrophages can induce a pro-inflammatory phenotype capable of Mtb containment and granuloma formation in the initial stages of infection, however over time $\mathrm{Mtb}$ alters this phenotype, generating a macrophage more permissible to Mtb growth (Huang et al., 2015; Refai et al., 2018). The mechanisms by which the macrophage is co-opted by Mtb to promote an environment amenable to its growth are not clearly understood. Unraveling the processes that mediate this transition from a predominantly pro-inflammatory macrophage population to a permissive one may provide new targets that can be therapeutically manipulated to promote bacterial clearance. Metabolic reprogramming has become recognized as a key cellular process that controls the responses of immune cells (Pearce and Pearce, 2013). Immune cells in different activation states preferentially induce different forms of metabolism to suit their energy requirements; classicallyactivated macrophages which can perform pro-inflammatory functions during infections vs. sustained regulatory functions carried out by alternatively-activated macrophages. Perhaps less appreciated, and what will be explored in this review, is the different metabolic intermediates generated that can act as signaling molecules and anti-microbial effectors during Mtb infection. These metabolic intermediates should be appreciated as important products in their own right, not merely by-products of the energetic demands of the cell.
It should also be noted that macrophage activation status is distinct from macrophage developmental origin, and that these factors overlay to define macrophage phenotype. The term macrophage encompasses a range of cells that can have different origins, phenotypes and functions. In recent years, it has become accepted that macrophages fall into two developmentally distinct populations-recruited and tissueresident macrophages (Ginhoux et al., 2010; Schulz et al., 2012). Recruited macrophages are of monocytic origin. Monocytes are derived from hematopoietic stem cell progenitors in the bone marrow which circulate in the peripheral blood until they migrate into tissues in response to growth factors, proinflammatory cytokines and microbial products (Epelman et al., 2014; Nourshargh and Alon, 2014). Tissue-resident macrophages however take up residency in specific tissues during embryonic development and proliferate locally, maintaining the population throughout the animal's lifespan (Guilliams et al., 2013; Yona et al., 2013; Epelman et al., 2014). Resident macrophages carry out homeostatic functions such as the clearance of cellular debris and processing of iron, as well as performing local immune surveillance (Davies et al., 2013). Tissue resident macrophages from different tissues are transcriptionally, thus likely functionally and metabolically, divergent (Gautier et al., 2012).

\section{Metabolic Heterogeneity in Lung Macrophages}

The lung compartment houses two ontologically distinct populations of macrophages-tissue resident alveolar macrophages (AM) and recruited interstitial macrophages (IM). Data from rodent models has suggested that AM are derived from fetal monocytes during lung development and proliferate locally to maintain the population in the lung (Guilliams et al., 2013), while IM are derived from circulating blood monocytes that are recruited to the interstitial space during infection (Huang et al., 2018). However, recent work by Byrne et al. has demonstrated that AM in the adult human lung are mostly peripheral in origin (Byrne et al., 2020). The authors used single-cell RNA-sequencing of the cells from the bronchoalveolar lavage (BAL) fluid of sex mis-matched lung transplant recipients to show that the majority of $\mathrm{AM}$ were recipient-derived, inferring that the AM population are replenished from circulating precursors in the periphery. Under homeostatic conditions, the AM population monitors the lung independently of monocyte-derived macrophages. AM are long-lived (Maus et al., 2006) tissue resident macrophages with a high phagocytic capability and are believed to be the primary initial immune cell to interact with Mtb (Cohen et al., 2018) and are therefore key in determining the subsequent immune response to Mtb infection.

Alveolar macrophages are unique in comparison to other tissue-resident macrophage populations in that they are in direct contact with the external environment, constantly being exposed to inhaled particulates, commensal bacteria and host-epithelial factors such as surfactant. The homeostatic activation state of AM has been controversial. A small population of IL-13-producing 
macrophages have been characterized in the lung compartment and this population increases in response to cigarette smoke, hinting that perhaps the normal population is more classically activated (Shaykhiev et al., 2009). Recent evidence indicates that $\mathrm{AM}$ are relatively plastic in homoeostatic conditions. A study which examined the lungs of 6 normal donors used immunohistochemistry to determine the activation states of the AM present found that healthy lung tissue AM expressed neither classical nor alternative activation markers (Bazzan et al., 2017). Interestingly, smoking and chronic obstructive pulmonary disease (COPD) increased the expression of both pro- and anti-inflammatory macrophage markers and the coexpression of these markers, highlighting that activation states do not have to be exclusive. The basal metabolic state of AM is believed to be distinct from that of peripherally derived macrophages. Gleeson et al. demonstrated using extracellular flux analyses that human AM are more reliant on oxidative phosphorylation than glycolysis and are metabolically similar to an alternatively activated monocyte-derived macrophage (MDM) (Gleeson et al., 2018). Their results also showed that while the basal metabolic state of the AM was more quiescent, significantly higher metabolic reserves were measured in the AM, indicating that perhaps AM are metabolically programmed so as to maintain an anti-inflammatory environment in the lung while having the capacity to mount a swift response to infection if required. Huang et al. compared the metabolic profile of IM and AM in a murine model of Mtb infection and found that IM adopt a glycolytic profile in response to infection while AM upregulate pathways involved in fatty acid oxidation (FAO) (Huang et al., 2018). They also showed that IM cultured ex vivo secreted more lactate than AM, indicating that even basally IM are more glycolytically active. AM reside in a lipid-rich environment, surrounded by pulmonary surfactant which is a phospholipid monolayer which lines the alveolar surfaces. AM have been shown to upregulate the scavenger receptor CD36 in response to Mtb infection, and this both increases the uptake of surfactant lipids and generates a phenotype more permissive to Mtb growth (Dodd et al., 2016). The lipid-centric environment and metabolism of AM may be exploited by Mtb to both fuel its growth and evade immune responses.

\section{Metabolic Flexibility in Immune Responses}

The notion that metabolic profiles underpin immune function derives from the clear relationship between macrophage activation state and metabolism. Under homeostatic conditions macrophages utilize mitochondrial oxidative phosphorylation to metabolize glucose and generate ATP. When macrophages become classically activated a metabolic shift occurs from oxidative phosphorylation toward glycolysis despite the availability of oxygen (Hard, 1970). Glycolysis converts glucose into pyruvate in the cytoplasm to produce ATP. During this glycolytic reprogramming, glucose preferentially generates lactate instead of pyruvate, and though glycolysis is less energy efficient, it can be quickly upregulated to allow rapid generation of cytoplasmic ATP. This is thought to meet the increased energy demands of activation. However, we know this metabolic reprogramming serves a further purpose than just meeting the ATP demand as cancer cells and T cells adopt Warburg metabolism to generate biosynthetic precursors to support cell division (O’Neill et al., 2016). However, macrophages (which are non-dividing) also adopt this metabolic profile. This glycolytic switch may be necessary due to the increased transcription and translation requirements of these cells which require the nucleotide building block ribose which is generated in the pentose phosphate pathway (PPP), a side branch of glycolysis; however blocking glycolysis has been shown to destroy the ability of the macrophage to contain Mtb growth (Gleeson et al., 2016). Moreover, Mtb has been shown to block macrophage metabolism to evade eradication by the immune system (Cumming et al., 2018; Hackett et al., 2020). We will now discuss these key metabolic pathways, the metabolites they generate and how they relate to Mtb infection, as summarized in Figure $\mathbf{1 .}$

\section{GLYCOLYSIS}

\section{Regulation of Glycolysis in Mtb-Infected Macrophages}

Glucose is transported into the cell through a glucose transporter, the primary rate-limiting glucose transporter in proinflammatory macrophages is GLUT1 (Freemerman et al., 2014), encoded by the SLC2A1 gene. GLUT1 is upregulated in response to $\mathrm{Mtb}$ infection to supply the cell with the glucose required to sustain the induction of glycolysis (Braverman et al., 2016). The first irreversible step of glycolysis is catalyzed by hexokinase $(\mathrm{HK})$ and is a rate limiting step, converting glucose to glucose6-phosphate. Though there are several isoforms of this enzyme, HK2 is the principal regulated form of this enzyme in most cell types (Wilson, 2003) and is inhibited by 2-DG and upregulated in response to Mtb infection (Braverman et al., 2016). Furthermore, it has been shown to be downregulated in diabetic patients who are associated with increased risk for Mtb infection ( $\mathrm{Qu}$ et al., 2012). At this point glucose-6-phosphate can continue down the pathway of glycolysis or be converted into glycogen or oxidized by the oxidative branch of the PPP (Gottlieb, 2011). The PPP is a parallel metabolic pathway that occurs alongside glycolysis, converting glucose-6-phosphate into NADPH which can be used in the generation of reactive oxygen and nitrogen species, and ribose-5-phosphate for the production of nucleotides. Activated macrophages have been shown to have increased PPP activity (Jha et al., 2015) and TLR stimulation suppresses carbohydratekinase like protein (CARKL), an inhibitor of the PPP pathway (Haschemi et al., 2012), although its impact on Mtb infection is unclear and has yet to be formally addressed. Cumming et al. have performed extracellular flux analysis and carbontracing experiments on human MDM infected with live virulent Mtb as well as Bacillus Calmette-Guérin (BCG) and dead Mtb which have indicated that live Mtb is able to suppress macrophage energy flux while less virulent and dead forms of Mtb drive glycolysis (Cumming et al., 2018). They showed that while dead Mtb enhanced the flux through the PPP, this was negatively regulated by live $\mathrm{Mtb}$, indicating that $\mathrm{Mtb}$ may have evolved mechanisms to restrict this pathway as an immune evasion mechanism. 


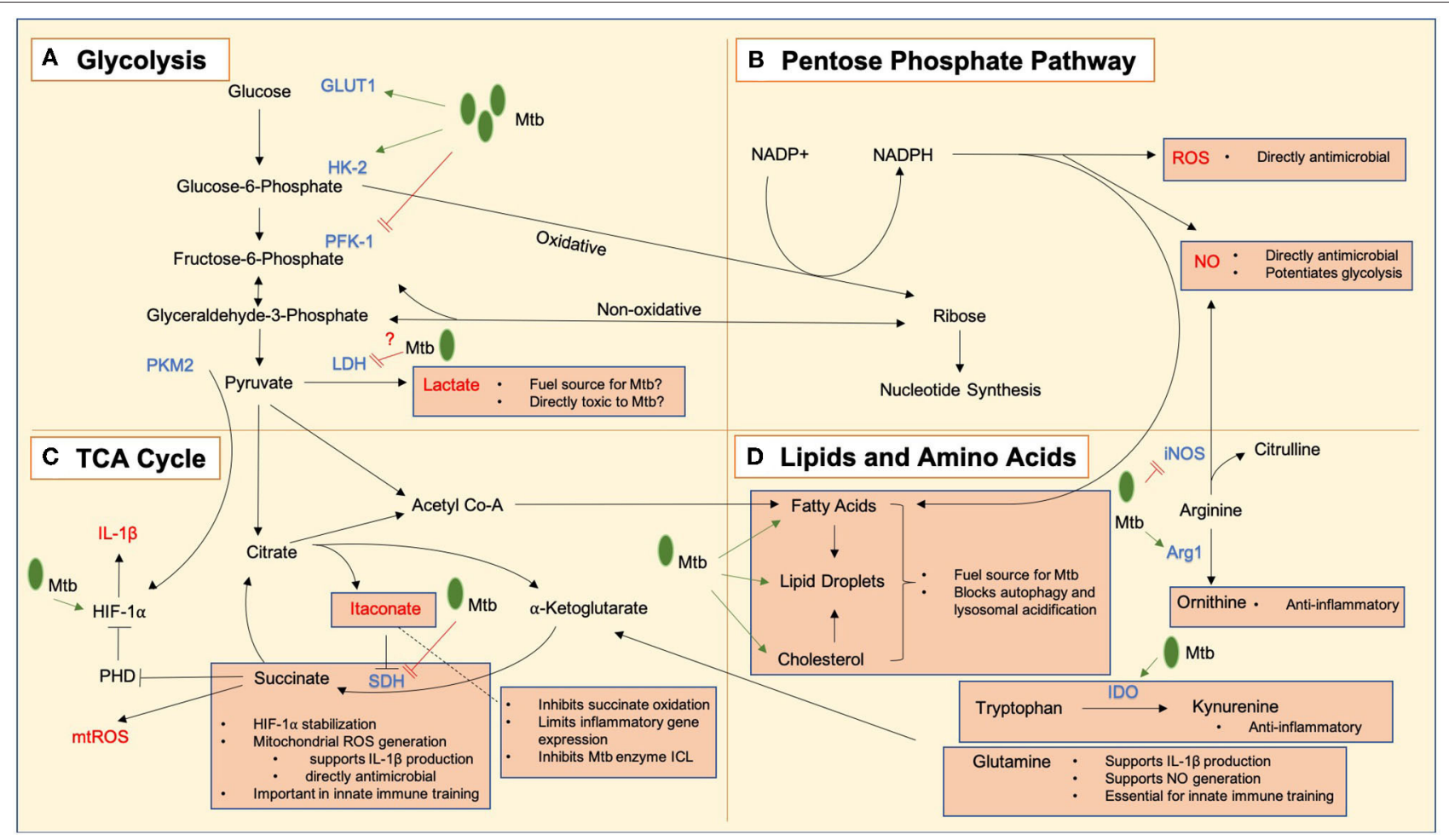

FIGURE 1 | Key metabolic pathways and metabolic intermediates in Mtb immune responses. Mycobacterium tuberculosis bacilli are notated as Mtb in green, and processes upregulated and downregulated by infection indicated using green arrows $(\rightarrow)$ and red lines $(=)$ respectively. (A) Glycolysis converts glucose to lactate which may act as both a fuel source for Mtb and have direct antimicrobial effects. GLUT1, the glucose transporter, is upregulated in response to Mtb infection, and hexokinase 2 (HK2) is also upregulated to allow an enhanced glycolytic rate. Mtb may limit the induction of glycolysis by negative regulation of the rate-limiting enzyme phosphofructokinase 1 (PFK-1). Lactate dehydrogenase (LDH) is upregulated in infected macrophages, allowing enhanced conversion of pyruvate to lactate, which may act as an alternative fuel source for Mtb or a directly toxic antimicrobial mediator. There is evidence that Mtb negatively regulates this process. Pyruvate kinase M2 (PKM2) works in tandem with hypoxia inducible factor 1 alpha (HIF-1 $\alpha$ ) to allow transcription of IL-1 $\beta$. (B) The pentose phosphate pathway produces NADPH which is used to generate reactive oxygen species (ROS) and nitric oxide (NO) which are directly antimicrobial, and NO has additional roles in the potentiation of glycolytic metabolism. Live Mtb may negatively regulate flux through this pathway to limit these actions. (C) A tricarboxylic acid (TCA) cycle break point leads to an accumulation of itaconate which can inhibit the Mtb enzyme isocitrate lyase (ICL), while also protecting the host from excessive inflammation by limiting the oxidation of succinate by succinate dehydrogenase (SDH) and inflammatory gene expression. A second TCA cycle break point leads to a build-up of succinate which can (i) lead to the generation of mitochondrial ROS (mtROS) which are directly antimicrobial and also support the production of IL-1 $1 \beta$, stabilize HIF- $1 \alpha$ to promote glycolysis by inhibiting prolyl hydroxylases (PHDs) and (iii) play a role in the induction of innate immune training. (D) Lipids are key cellular fuel sources exploited by Mtb to promote its growth and inhibit its destruction within the cell. Amino acids are also important in Mtb responses. Arginine can be metabolized into antimicrobial NO by inducible nitric oxide synthase (iNOS) or into ornithine by arginase 1 (Arg1) which is anti-inflammatory. Likewise, tryptophan can be broken down into kynurenine by indoleamine 2,3-dioxygenase (IDO) which is also anti-inflammatory. Glutamine has been shown to play roles in the production of IL-1 $1 \beta$, generating NO and inducing innate immune training.

\section{Impact of Host Glycolysis on Macrophage Mtb Responses}

Pro-inflammatory macrophages have been shown to induce glycolysis to meet increased energy demands and provide biosynthetic precursors to promote antimicrobial responses during inflammation (Tannahill et al., 2013). The induction of glycolysis is orchestrated by the transcription factor hypoxia-inducible factor 1 (HIF-1) which acts as a master regulator of pro-inflammatory immune functions (Palazon et al., 2014). HIF-1 has a large number of target genes, including the transporters and enzymes which constitute the glycolytic machinery (e.g., GLUT1, HK2, LDHA), but also many pro-inflammatory cytokines, chief of which is IL-1 $\beta$.
The molecular mechanisms by which this metabolic switch to glycolysis in response to infection is mediated are beginning to be unraveled.

Pyruvate kinase M2 (PKM2) is a splice variant of the glycolytic enzyme pyruvate kinase, which was originally identified as being upregulated in cancer to enable Warburg metabolism (Christofk et al., 2008). Palsson-McDermott et al. identified PKM2 as a key metabolic regulator which mediates HIF- $1 \alpha$ activation in LPS-stimulated macrophages (Palsson-McDermott et al., 2015). They showed that LPS induced PKM2 expression and this mediated the binding of PKM2 and HIF- $1 \alpha$ to a hypoxia response element (HRE) in the promoter of the gene encoding IL-1 $\beta$. The authors additionally showed that this PKM2/HIF-1 $\alpha /$ IL- $1 \beta$ 
axis was important for containing Mtb infection in an in vitro murine model.

IL-1 $\beta$ has been implicated as one of the most important cytokines for containing Mtb infection. Mayer-Barber et al. demonstrated that IL- $1 \beta$ induces the expression of eicosanoids which limit excessive type I interferon signaling and promote bacterial containment (Mayer-Barber and Sher, 2015). Braverman et al. have shown that HIF-1 $\alpha$ is an essential gene for the control of $\mathrm{Mtb}$ infection and additionally that the macrophage-activating cytokine interferon- $\gamma$ (IFN- $\gamma$ ) acts through HIF- $1 \alpha$ to induce glycolytic reprogramming and promote bacterial containment (Braverman et al., 2016). Gleeson et al. went on to show that Mtb infection induces an immunometabolic shift to glycolysis in several macrophage models including human alveolar macrophages and that this glycolytic response is essential for sufficient induction of IL- $1 \beta$ and control of Mtb growth (Gleeson et al., 2016). Our work has shown that Mtb negatively regulates this induction of glycolysis over time by limiting the induction of an isoform of the glycolytic rate-limiting enzyme phosphofructokinase 1 (PFK-1) (Hackett et al., 2020). PFK-1 is a tetrameric enzyme that can be composed of different combinations of the muscle $(M)$, platelet (P) and liver (L) isoforms of the enzyme. Each isoform is encoded by a different gene and isoforms are differentially expressed in different tissues. RNA-seq analysis of murine BMDM showed that PFK-L and PFK-P are upregulated 24 hours post Mtb infection, however PFK-M is not (Shi et al., 2015), and our work showed that Mtb induced microRNA-21 which directly limited PFK-M expression and consequently dampened glycolytic induction and antimicrobial responses. MicroRNA have emerged as key molecules involved in regulating a range of cellular processes, including innate immunity (Momen-Heravi and Bala, 2018). In addition to miR-21, other microRNA species have been implicated in the pathogenesis of $\mathrm{Mtb}$ infection, reviewed by Behrouzi et al. (2019). For example, miR-33 has been demonstrated to be induced by Mtb infection and limit lipid metabolism and autophagy thus promoting Mtb survival (Ouimet et al., 2016). Another recent study has demonstrated that multi-drug-resistant strains of Mtb modulate macrophage metabolism to limit IL-1 $\beta$ responses (Howard et al., 2018). Together these observations underline how critical adequate macrophage glycolytic activation is to Mtb immunity, and how Mtb has evolved mechanisms to limit metabolism and evade the immune response.

Although it is well established that fatty acids are a key source of energy for Mtb which feed central carbon metabolism (McKinney et al., 2000; Marrero et al., 2010), macrophage metabolic reprogramming may alter the availability of these lipids, and instead other carbon sources in the Mtb microenvironment can also be utilized. Lactate dehydrogenase $(\mathrm{LDH})$ catalyzes the interconversion of pyruvate and lactate and accompanying interconversion of NADH and NAD+. LDHA is upregulated in response to metabolic reprogramming in macrophages and dendritic cells (Kelly and O'Neill, 2015; Braverman et al., 2016). Accumulation of lactate, the final product of glycolysis, is enhanced in response to increased rates of glycolysis and can be used as a surrogate marker of glycolytic activity (Rogatzki et al., 2015). The view of lactate as a waste product of metabolism has begun to be re-examined. Lactate has been shown to inhibit T cell migration, polarize tumor-associated macrophages toward an M2-phenotype (Colegio et al., 2014), inhibit pro-inflammatory macrophage responses and glycolytic programming and drive dendritic cells toward a more tolerogenic phenotype (Errea et al., 2016). Lactate has also recently been reported to facilitate $\mathrm{Mtb}$ growth by acting as an additional carbon source (Billig et al., 2017). Lactate has been shown to be present in significant quantities at the site of infection and the granuloma (Shin et al., 2011; Somashekar et al., 2011; Shi et al., 2015), both intra- and extracellularly (Serafini et al., 2019). Billig et al. showed that Mtb can use lactate as its sole carbon source in vitro (Billig et al., 2017), though at higher concentrations it was found to be toxic to the bacterium. Interestingly, a mutant Mtb strain that lacked the required lactate dehydrogenase gene (Lld2) to process lactate showed sensitivity to the toxic effects of lactate even at lower concentrations, indicating that perhaps lactate is used as a fuel by Mtb not only because of its availability but also to remove it from its environment. Lactate has been shown to inhibit the growth of other bacteria through the generation of reactive oxygen species (ROS) (Abbott et al., 2009), thus the oxidation of lactate to pyruvate by Mtb may be both for fuel and protective purposes. A study which compared two clinical isolates of $\mathrm{Mtb}$ found that in a lipid-poor environment, one of the strains upregulated Lld2, further indicating a role for lactate as a substitute fuel source (Baena et al., 2019). Additionally, genome analysis of lineage $4 \mathrm{Mtb}$ genomes (the most common and globally distributed Mtb lineage) identified several mutations in the promoter and protein coding regions of Lld2 which had independently arisen over a hundred times (Brynildsrud et al., 2018). The codon mutations were then further identified in other Mtb lineages and associated with a significant positive effect on transmissibility.

More evidence for the alternative role of lactate as a molecule upregulated by the macrophage to combat $\mathrm{Mtb}$ infection comes from the finding that infected macrophages upregulate LDH-A through HIF-1 $\alpha$ (Osada-Oka et al., 2019), allowing increased conversion of pyruvate to lactate. HIF-1 $\alpha$-deficient macrophages were found to have significantly higher levels of intracellular pyruvate, and LDH-A-deficient macrophages were not as proficient at containing Mtb growth as wild-type cells. This study indicated that pyruvate is the preferred intracellular carbon source over glucose, thus the upregulation of LDH in response to $\mathrm{Mtb}$ infection may be a defense mechanism to both deprive the bacterium of fuel and boost anti-mycobacterial ROS generation. De Carvalho et al. recently demonstrated that pyruvate and lactate are in fact superior carbon sources for Mtb than glucose and fatty acids but only when oxygen is plentiful (Serafini et al., 2019). Recent evidence from Cumming et al. shows that while lactate and pyruvate are both enriched in the supernatant fluid of human monocyte-derived macrophages infected with BCG and dead Mtb, live virulent Mtb decreased the production of these metabolites, indicating a negative regulation of this process to aid in immune evasion (Cumming et al., 2018). Our work found that neither dead nor live virulent Mtb induced LDHA in human alveolar macrophages (Hackett et al., 2020), and 
together these findings indicate that the glycolysis/LDHA/lactate axis is an important antimicrobial mechanism which Mtb actively suppresses.

\section{KREBS CYCLE INTERMEDIATES}

The Krebs cycle is becoming increasingly recognized as a central regulatory component of the immunometabolic programme. Also known as the tricarboxylic acid (TCA) and citric acid cycle, immunologists are reconsidering this process as more than a way of generating energy but rather as a pivotal system through which metabolites which can regulate the immune response are generated. LPS-activated macrophages have been demonstrated to suppress oxidative phosphorylation through the Krebs cycle and to accumulate the intermediates succinate and itaconate (Tannahill et al., 2013). It is hypothesized that there are two metabolic "breaks" in the cycle which occur in response to LPS stimulation. The first breakpoint occurs at the third step of the cycle where isocitrate dehydrogenase (IDH) converts isocitrate to alpha-ketoglutarate. Activated macrophages downregulate Idh mRNA expression to limit this conversion, with an associated accumulation of itaconate (Tannahill et al., 2013). The second breakpoint is thought to occur in the final step of the cycle at complex II of the electron transport chain, succinate dehydrogenase (SDH), where fumarate is converted to succinate and an accumulation of succinate occurs (Jha et al., 2015). However, whether these TCA cycle breakpoints occur in the case of Mtb infection remains to be verified, the impact of these metabolites in Mtb responses is important.

\section{Succinate}

In recent years, succinate has begun to be appreciated as a metabolite that accumulates in pro-inflammatory macrophages and functions as an immune signal (Tannahill et al., 2013). Succinate can play several roles in amplifying the immune response. Tannahill et al. observed that succinate accumulates following macrophage activation and signals through HIF- $1 \alpha$ to induce IL-1 $\beta$ production (Tannahill et al., 2013). While NFKB is thought to be responsible for the majority of the early induction of IL- $1 \beta$, HIF- $1 \alpha$ can also induce IL- 1 transcription with both human and murine IL-1 $\beta$ genes containing HIF binding sites (Fang et al., 2009; Tannahill et al., 2013), and this is thought to be responsible for the sustained induction of IL-1 later in the course of inflammation In the inflammatory macrophage, HIF- $1 \alpha$ can also be stabilized even in normoxia by the elevated levels of succinate which inhibits PHDs (Tannahill et al., 2013), allowing IL-1 $\beta$ transcription to occur. This succinatemediated stabilization of HIF-1 $\alpha$ works in tandem with the PKM2-mediated trans-activation already discussed (PalssonMcDermott et al., 2015).

Oxidation of succinate has additionally been shown to play a key role in the inflammatory process, allowing the Krebs cycle to serve as a ROS generation system (Mills et al., 2016). Both succinate oxidation by SDH and increased mitochondrial membrane potential are required for ROS generation which is in turn required for the HIF-1 $\alpha$ dependent potentiation of IL$1 \beta$ signaling (Tannahill et al., 2013). An in vitro model where
SDH was inhibited in mice which were injected with LPS showed that this $\mathrm{SDH}$ inhibition reduced inflammation, decreasing the production of the pro-inflammatory cytokines TNF- $\alpha$ and IL- $1 \beta$ and enhancing the production of anti-inflammatory IL-10 (Mills et al., 2016). Garaude et al. have additionally shown that live E. coli bacteria (but not dead bacteria) are able to alter the assembly of electron transport chain supercomplexes which contributed to anti-bacterial responses (Garaude et al., 2016). These studies highlight the importance of mitochondrial ROS as an antimicrobial signal. In the case of Mtb infection, while the glycolytic machinery is upregulated, there is a concomitant decrease in the expression of Krebs cycle genes including SDH subunits SDHA, SDHC and SDHD, which would likely contribute to succinate accumulation (Shi et al., 2019). Murine lungs infected with Mtb have been shown to have an accumulation of succinate (Shin et al., 2011) indicating that this metabolic break is of functional importance in Mtb in vivo infection, however the Mtb model has not been specifically looked at in this context. Cumming et al. found increased flux in succinate production in response to BCG infection of human MDM, however this was not observed for Mtb (Cumming et al., 2018). Given that SDH upregulation has been shown to be important for ROS generation and HIF-1 $\alpha$ stabilization, both of which have been demonstrated to play a role in $\mathrm{Mtb}$ responses, it would be interesting to examine the dynamic contributions of succinate and SDH in Mtb infection.

Succinate has also been shown to bind a G-protein coupled receptor now known as SUCNR1 (He et al., 2004). Following activation of the macrophage with LPS, succinate accumulates and SUCNR1 expression increases, and succinate signaling leads to an enhanced IL-1 $\beta$ response (Littlewood-Evans et al., 2016). IL-1 $\beta$ in turn enhances SUCNR1 and thus a positive feedback loop occurs. More recently, Keiran et al. have demonstrated a role for succinate/SUCNR1 signaling in promoting an antiinflammatory phenotype in adipose tissue resident macrophages and protected the host from tissue inflammation both in homeostasis and in metabolic stress (Keiran et al., 2019). These confounding results may suggest the role of succinate in SUCNR1 signaling may be tissue or context specific. The role of SUCNR1 in $\mathrm{Mtb}$ infection has yet to be established, however given that succinate accumulates in Mtb infection and SUCRN1 is implicated in determining macrophage phenotype in the context of inflammation, its role in Mtb infection may be important.

\section{Itaconate}

$\mathrm{SDH}$ activity is in turn regulated by another metabolite itaconate. Immune-responsive gene 1 (Irg1) produces itaconate from the Krebs cycle intermediate aconitate (Michelucci et al., 2013), and this itaconate was shown to inhibit the growth of microorganisms including Mtb in liquid culture. Irg1 expression and thus levels of itaconate are increased following macrophage stimulation with LPS (Strelko et al., 2011). Itaconate was shown to inhibit SDH-mediated oxidation of succinate and thus limit pro-inflammatory responses (Lampropoulou et al., 2016). Itaconate has additionally been shown to limit inflammation by activation of the transcription factor Nrf2 (Mills et al., 2018) which limits inflammatory gene expression and downregulates 
type 1 interferons. Itaconate has been measured in the Mtbinfected murine lung (Shi et al., 2019), while Irg1 has been shown to be highly upregulated in the murine macrophage and lung after Mtb challenge (Kang et al., 2011).

As well as limiting the oxidation of succinate and activating Nrf2, itaconate inhibits the activity of the microbial enzyme isocitrate lyase (ICL1) (Williams et al., 1971). ICL1 is part of the glyoxylate shunt which is thought to be an adaptation to low-glucose environments such as the phagolysosome (Luan and Medzhitov, 2016) allowing the bacterium to be fueled by 2carbon compounds (Lorenz and Fink, 2002). ICL1 has shown to be upregulated in phagocytosed Mtb bacilli (Graham and Clark-Curtiss, 1999) and has shown to be required for longterm persistence of Mtb in a murine infection model (McKinney et al., 2000; Munoz-Elias and McKinney, 2005). Wang et al. identified an enzyme in the virulent Mtb strain H37Rv which is capable of degrading itaconate (Wang et al., 2019), deletion of which reduced the bacterial burden in a murine model of infection. Irg1 has been shown to be essential for host survival in murine Mtb infection, with Irg1 knockout mice exhibiting excessive inflammation when infected with Mtb and a higher bacterial burden (Nair et al., 2018). Nair et al. also carried out this experiment with a strain of Mtb in which icl1 had been deleted. Irg1 knockout mice were still unable to control Mtb infection and were killed by the infection, thus $\operatorname{Irg} 1$ and itaconate likely control Mtb growth in vivo independently of the inhibitory effect on ICL1 that has been noted in vitro. Likely the combined effects of succinate-driven pro-inflammatory effector mechanisms and itaconate-driven resolving mechanisms attempt to clear $\mathrm{Mtb}$ infection while minimizing damage to the host tissue. In line with this postulation is the recent findings of a metabolomic study of mouse lung following Mtb infection which noted an increase in succinate 4 weeks post-infection which was dramatically reduced by week 9 of infection, while itaconate steadily accumulated throughout this time course (Fernandez-Garcia et al., 2020).

\section{Regulation or Response: The Causality Dilemma}

It should be noted that our understanding of these events may be about to shift. Recent findings by Palmieri et al. have identified nitric oxide (NO) as a mediator of Krebs cycle changes during the inflammatory response (Palmieri et al., 2020) and indicated that this model of a "break" in the Krebs cycle leading to succinate, itaconate and citrate accumulation and thus metabolic reprogramming of the cell may need revision. The authors found that NO directly mediated the metabolic reprogramming through aconitase 2 and $\mathrm{PDH}$, rather than being a downstream result of rewiring. Braverman et al. have previously found that $\mathrm{NO}$ is required for HIF- $1 \alpha$ stabilization during Mtb infection and negatively regulates $\mathrm{NF} \kappa \mathrm{B}$ signaling to limit inflammation (Braverman and Stanley, 2017). In the case of the repurposing of the TCA cycle to generate ROS and immunometabolites it is yet to be determined whether impaired glycolysis and thus a reduced availability of pyruvate to feed flux through the TCA cycle is the initial event in reprogramming, or rather a break in the TCA cycle and accumulation of intermediates which causes an upregulation in glycolysis. In the context of Mtb infection, this is even less clear cut, with some reports finding oxidative phosphorylation intact (Hackett et al., 2020), perhaps being fueled by alternative pathways such as intracellular lipids liberated by FAO (Knight et al., 2018).

\section{FAO AND LIPID METABOLISM}

When examining the relationship between the immune response to Mtb infection and metabolism, the fuel preferences of both the macrophage and the bacterium, and the consequent impacts of the by-products of the utilization of these fuels by both species must be taken into account. In the context of Mtb infection, murine IM and AM have been shown to be metabolically and functionally distinct. Huang et al. have shown using fluorescent Mtb reporter strains that infected IM adopt a pro-inflammatory, glycolytic phenotype which produces IL-1 $\beta$ and NO and are better at clearing the invading pathogen, while AM are more reliant on FAO, produce type 1 interferons and provide a more permissive environment for Mtb growth (Huang et al., 2018). Mtb requires a carbon fuel source in order to perform its metabolism, and lipids and fatty acids have long been recognized as its preferred energy source. The dominating FAO metabolism of the AM induced by Mtb infection creates a nourishing, permissive environment for the bacterium to replicate.

Mtb uses host lipids (fatty acids and cholesterol) for its optimal colonization of the host. Cholesterol import by Mtb as a source of carbon has been shown to be essential for long-term infection of a murine host and for replication inside activated macrophages (Pandey and Sassetti, 2008). Aberrant cholesterol status has been linked to poorer Mtb responses-hypercholesterolemia has been correlated with Mtb risk in a human study (Soh et al., 2016), while apoE-/- hypercholesteremic mice were shown to have a higher bacterial burden and more severe lung damage (Martens et al., 2008). Cholesterol accumulation has been linked to inhibition of phagosomal maturation (Huynh et al., 2008), a clear advantage to $\mathrm{Mtb}$, as well as impaired autophagy and thus less bacterial killing (Chandra and Kumar, 2016). Oxidized low density lipoprotein (oxLDL) has also been shown to accumulate in granulomas and have been associated with increased Mtb growth in an in vivo guinea pig model (Palanisamy et al., 2012). oxLDL is resistant to lipolysis and encourages lipid accumulation within the macrophage and poor efflux of cholesterol (Brown et al., 2000). Vrieling et al. have recently linked oxLDL and impaired macrophage responses to $\mathrm{Mtb}$ and proposed this as a contributing element to the increased risk of Mtb infection in type 2 diabetics (Vrieling et al., 2019b). They measured higher plasma oxLDL in diabetic patients and showed that in vitro oxLDL treatment of human macrophages significantly increased the bacterial burden by inducing cholesterol accumulation and a lysosomal dysfunction which impaired lysosome localization with Mtb.

Fatty acids are also plentiful in the macrophage, particularly as the granuloma forms and matures (Kim et al., 2010) and are utilized by Mtb as a source of lipid building blocks (Lee et al., 2013). In the case of Mtb, foamy macrophage generation 
can be induced by infection (D'Avila et al., 2006), and these lipid-rich macrophages are present in high numbers in the Mtb granuloma (Peyron et al., 2008). Foamy macrophages are generated when macrophage lipid intake and export are unbalanced and an accumulation of lipoproteins occurs, and this phenotype has been associated with other diseases, particularly atherosclerosis (Moore et al., 2013). While atherosclerotic foam cells are cholesterol-dominated, Mtb granulomas are richest in triglycerides (Guerrini et al., 2018), with mycobacterial ligands signaling through macrophage receptors to alter triglyceride content (Dkhar et al., 2014). Geurrini et al. have shown in vitro that this lipid droplet formation in response to $\mathrm{Mtb}$ infection is driven by signaling through the TNF receptor which activates caspases and mTORC1 (Guerrini et al., 2018). The exact mechanism of this has yet to be untangled but in other models peroxisome proliferator-activated receptor- $\gamma$ (PPAR- $\gamma$ ) has been shown to promote lipogenesis (Li et al., 2014), and this nuclear receptor has been implicated in regulation of the link between macrophage lipid metabolism and foam cell generation in Mtb infection. PPAR- $\gamma$ is highly expressed in alveolar macrophages (Schneider et al., 2014), the primary host cell for Mtb. Almeida et al. demonstrated that murine macrophages infected with BCG upregulate PPAR- $\gamma$ in a TLR2 dependent manner which enhanced lipid body formation and PGE2 synthesis (Almeida et al., 2009), while Rajaram et al. have described PPAR- $\gamma$ activation following Mtb phagocytosis in human macrophages which suppressed pro-inflammatory responses and enhanced Mtb growth (Rajaram et al., 2010). Guirado et al. further demonstrated that PPAR- $\gamma$ negatively regulates macrophage activity and impairs Mtb responses in an in vivo murine model (Guirado et al., 2018). Interestingly, the essential vitamin $\mathrm{B} 1$ has been shown to enhance macrophage Mtb responses in a murine in vivo model by limiting PPAR- $\gamma$ activation (Hu et al., 2018).

The induction of the foamy macrophage phenotype has been viewed as a mechanism by which Mtb can gain fuel and carbon building blocks from its host, however there is accumulating evidence that the induction of this phenotype may serve the additional purpose of blocking antimicrobial responses. Virulent strains of Mtb have been shown to induce the foamy macrophage phenotype and this blocks autophagy and lysosomal acidification (Singh et al., 2012). Cumming et al. demonstrated that live Mtb increased macrophage dependency on exogenous fatty acids which would likely encourage lipid droplet formation, while dead Mtb did not have the same effect (Cumming et al., 2018). Providing an alternative view of lipid droplet formation is the study from Knight et al. in which they have shown that Mtb droplet formation is not driven by the bacterium, but rather the host inflammatory response (Knight et al., 2018). Using a murine model they have shown that lipid droplet formation requires IFN- $\gamma$ and HIF- $1 \alpha$, and this in turn is required for the production of PGE2 and leukotriene B4 which are protective in Mtb infection (Mayer-Barber and Sher, 2015), as well as being a method by which lipids are sequestered from Mtb. These findings challenge the current paradigm somewhat but still emphasize that fatty acid metabolism is a key process modulated by both the host and $\mathrm{Mtb}$ - the macrophage and the bacterium are in a metabolic arms race. Metabolic reprogramming may explain the formation of lipid droplets in response to Mtb via increased flux through other metabolic pathways such as the PPP. Our work has shown that Mtb negatively regulates the activity of PFK-1 (Hackett et al., 2020), a key rate-limiting enzyme in glycolysis at which glucose derivatives can continue through glycolysis or be shuttled into the PPP which could fuel fatty acid synthesis. This targeting of glycolysis may aid in immune evasion by limiting glycolysismediated antimicrobial activities while simultaneously boosting the production of fatty acids and nucleotides.

\section{AMINO ACIDS}

As well as serving as the biological building blocks of proteins, amino acids and metabolites derived from them are also able to act as direct anti-mycobacterial agents (Qualls and Murray, 2016). Two amino acids which are associated with the alternatively activated macrophage, tryptophan and arginine, are also implicated in Mtb infection.

\section{Tryptophan}

Unlike bacteria, animals are unable to synthesize tryptophan and this essential amino acid which is required for a broad array of biological functions must be obtained in the diet. While tryptophan is known to have roles such as being the precursor to serotonin, the role of tryptophan catabolism through the kynurenine pathway is of importance to immune function (Moffett and Namboodiri, 2003). Indoleamine 2,3-dioxygenase 1 (IDO1), the enzyme which catalyzes the catabolism of tryptophan, has been found to be significantly increased in the Mtb granuloma in non-human primate models (Mehra et al., 2013) and has been shown to be an effective biomarker for active tuberculosis infection (Adu-Gyamfi et al., 2017). IDO1 plays an inhibitory role in inflammation by limiting the activity of CD4+ $\mathrm{T}$ cells, and its catabolism of tryptophan has been shown to be essential in mediating tolerance (Munn et al., 1998) both through tryptophan depletion and the accumulation of kynurenines, tryptophan metabolites which have an immunosuppressive effect (Belladonna et al., 2007). Tryptophan metabolites have also been shown to promote TGF- $\beta$ production by dendritic cells leading to the generation of regulatory T cells (Yan et al., 2010).

For other intracellular bacterial infections such as chlamydia, induction of IDO in the macrophage by IFN $-\gamma$ provided by CD4+ $\mathrm{T}$ cells serves to deprive the pathogen of tryptophan (Byrne et al., 1986; Beatty et al., 1994), however Mtb can synthesize tryptophan de novo and thus tryptophan depletion is not the mechanism of protection in the context of $\mathrm{Mtb}$ infection (Zhang et al., 2013). Inhibiting Mtb tryptophan synthesis using a small molecule inhibitor to effectively make the bacterium a tryptophan auxotroph has been shown to improve Mtb containment (Zhang et al., 2013). IDO1 activation in Mtb infection has in fact been associated with poorer outcomes in animal models (Foreman et al., 2016) and higher serum IDO1 activity (and therefore lower tryptophan, higher kynurenine concentration) has been associated with a worse prognosis in human patients (Suzuki et al., 2012). IDO1 inhibition has 
been shown to improve mycobacterial containment in a nonhuman primate model of Mtb infection, enhancing CD4+ T cell penetration into the granuloma (Gautam et al., 2018). Given that tryptophan is not synthesized by the human host, enzymes in the Mtb tryptophan synthesis pathway could be potential targets for novel treatments.

\section{Arginine}

Reactive nitrogen species are potent anti-microbial effectors and signaling molecules (Braverman and Stanley, 2017). Arginine can be metabolized by macrophage nitric oxide synthase (NOS) to produce citrulline and NO. NO can be further metabolized into reactive nitrogen species, including nitrite, which can act as antimicrobial effectors against Mtb, as well as inducing glycolytic reprogramming in response to infection through stabilization of HIF- $1 \alpha$ and acting in a resolving capacity by limiting NFKB signaling (Braverman and Stanley, 2017). Inducible nitric oxide synthase (iNOS) is encoded by the NOS2 gene and is the isoform of this enzyme that can be upregulated upon inflammatory activation and can function independently of calcium, unlike the constitutive isoforms. Conversely, arginase 1, the cytoplasmic isoform of arginase which Mtb is known to specifically drive in infection, inhibits NO synthesis through several proposed mechanisms including competing with NOS for arginine as a substrate. Arginase-1 converts arginine into urea and ornithine from which hydroxyproline and polyamines can be generated for wound-healing (Mills et al., 2000). Polyamines themselves can also inhibit iNOS activity (Southan et al., 1994). Arginase-1 is upregulated downstream of TLR and cytokine-signaling and can reduce iNOS activity and impair the production of nitrite species (El Kasmi et al., 2008). The balance between these enzymes is important in determining the fate of arginine in the macrophage and thus its potential to produce anti-microbial nitrogen species.

Expression of arginase 1 characterizes the alternatively activated macrophage (Byers and Holtzman, 2011) and is associated with a reduced propensity for bacterial clearance (El Kasmi et al., 2008). The iNOS/arginase-1 macrophage activation state paradigm is more clearly defined in the murine model, while signals in the human model that drive macrophage activation remain much more elusive, suggesting that macrophages in human Mtb infection fall on a spectrum of activation and skewing the population toward either end of this spectrum is what determines infection outcome. Non-human primates infected with Mtb have been shown to have both iNOSand arginase-1-expressing macrophages in granulomas, with pro-inflammatory iNOS-positive macrophages organized at the center of the granuloma surrounded by alternatively activated arginase 1-positive macrophages on the periphery, and this distribution is mirrored in human granulomas (Mattila et al., 2013).

To replace the depleted arginine in the macrophage, another amino acid, citrulline, can be converted to arginine ( $\mathrm{Wu}$ and Brosnan, 1992) by the enzyme argininosuccinate synthase (Ass1). Citrulline has been shown to accumulate in murine lungs during the course of Mtb infection, coinciding with the upregulated expression of Ass1 in myeloid cells, deletion of which increased bacterial burden (Lange et al., 2019). Furthermore, this arginine synthesized from citrulline has been shown to be used effectively by iNOS but is less susceptible to arginase 1 depletion than imported arginine (Rapovy et al., 2015). Low plasma citrulline concentration has been observed in patients with active Mtb disease (Weiner et al., 2012), with the ratio of citrulline to arginine being able to distinguish patient samples from controls (Vrieling et al., 2019a). Thus, while arginine supplementation as a therapeutic strategy has had mixed reported efficacy (Schon et al., 2003; Ralph et al., 2013), perhaps citrulline supplementation may hold some future therapeutic potential, having also been shown to aid in $\mathrm{CD} 4+\mathrm{T}$ cell accumulation and activation in a murine Mtb infection model (Lange et al., 2017).

Arginine regeneration from citrulline may also be linked to another metabolic process, the Krebs cycle. The Krebs cycle of activated macrophages is downregulated due to decreased delivery of pyruvate and becomes functionally broken in two places leading to an accumulation of citrate (Infantino et al., 2011) and succinate (Jha et al., 2015). During this inflammatory activation, an argininosuccinate shunt is engaged which bridges the urea cycle and the Krebs cycle (Jha et al., 2015), thus potentially both generating fumarate for the Krebs cycle and arginine for NO production through Ass1 (Murray, 2016).

Recently Yurdagul et al. described a novel role for arginine whereby arginine and ornithine from apoptotic cells phagocytosed by macrophages is metabolized to putrescine by Arg1 and ornithine decarboxylase (ODC) to promote continued efferocytosis through Rac1 activation (Yurdagul et al., 2020). Cell death is also a key process in the interaction between Mtb and the host. Mtb infection can induce necrotic cell death whereby the infected cell lyses and allows further spread of the bacilli, which is favorable for Mtb. The Mtb ESX1 secretion system has been shown to be the molecular driver behind this promotion of necrotic cell death and its absence partly responsible for the attenuation of the strain of Mycobacterium bovis used in the BCG vaccine (Pym et al., 2002). Alternatively, apoptosis can be instigated, a controlled death program that maintains the integrity of the cell membrane and reduces Mtb survival (Behar et al., 2011). Infection with Mtb has been shown not only to induce macrophage apoptosis, but also to induce apoptosis of neighboring uninfected macrophages (Kelly et al., 2008). This bystander apoptosis may limit Mtb survival by depriving it of its host cell. More virulent strains of Mtb have developed resistance mechanisms to combat this by blocking apoptosis (Velmurugan et al., 2007). Apoptosis in and of itself is not effective at killing Mtb, however the process of efferocytosis of infected apoptotic macrophages has been demonstrated to be an important mechanism for promoting bacterial clearance (Martin et al., 2012). It would be interesting to investigate whether the arginine/Rac1 mechanism of promoting continued efferocytosis is relevant in the case of Mtb infection.

\section{Glutamine}

Like fumarate, glutamine can also be used as an alternative carbon fuel for the Krebs cycle, and a source of citrate in fatty acid synthesis. Glutamine plays many roles in facilitating immune function. Activated macrophages increase glutamine uptake, and it has long been known to be required for the production of 
IL-1 $\beta$ by LPS-activated macrophages (Wallace and Keast, 1992). Macrophages from mice fed on a glutamine enriched diet were shown to produce more TNF- $\alpha$, IL- $1 \beta$ and IL-6 in response to LPS stimulation (Wells et al., 1999). Glutamine has also been shown to play a role in nitric oxide production (Murphy and Newsholme, 1998), replenishing intermediates in the nitrite and urea cycles to maintain flux through the system. More recently, glutamine been shown to have an essential role in alternative activation of macrophages, with glutamine deprivation and consequent effects on the TCA cycle preventing polarization (Jha et al., 2015; Palmieri et al., 2017). Activation of mTOR can be mediated by glutamine, and thus glutamine can play a role in the induction of autophagy through mTORC1 (He et al., 2016).

Several pathogens have been shown to alter glutamine metabolism. A recent study showed that infection of macrophages with Leishmania donovani increased expression of genes involved in glutamine metabolism, and the inhibition of glutaminolysis increased susceptibility to infection and was associated with a more anti-inflammatory recruited myeloid population and poorer T-cell mediated responses (Ferreira et al., 2020). Cumming et al. have shown that Mtb infection creates a dependency on glutamine in infected human MDM (Cumming et al., 2018). Koeken et al. have further explored the importance of glutamine in the case of Mtb infection (Koeken et al., 2019). They showed that the glutamine transcriptome is significantly upregulated in macrophages in response to $\mathrm{Mtb}$ infection, both in vitro and in vivo, and that interference with either glutamine supply or its catabolism decreased cytokine responses to $\mathrm{Mtb}$ infection, particularly IL-1 $\beta$. Additionally, they identified single nucleotide polymorphisms (SNPs) in genes belonging to the glutamine pathway that altered cytokine responses to Mtb in human peripheral blood mononuclear cells (PBMCs). Together these findings indicate an important role for glutamine in a robust response to Mtb infection, though the role for glutamine in an in vivo infection has yet to be tested. Glutamine has additionally been shown to be essential in the induction of innate immune training (Arts et al., 2016), discussed below.

\section{METABOLIC REWIRING TO PRIME MTB RESPONSES}

Innate immune training or trained immunity is the concept that innate myeloid cells can mount a better immune response to a secondary exposure to a non-specific insult or pathogen (Netea et al., 2011). The induction of this trained phenotype in response to a range of stimuli including the metabolite oxLDL, the fungal cell wall component $\beta$-glucan and whole microbes such as BCG (van der Heijden et al., 2018), is dependent on immunometabolic reprogramming which mediates epigenetic changes (Kleinnijenhuis et al., 2012; Cheng et al., 2014). A number of metabolic pathways and metabolites are being shown to have key roles in the mediation of training. For example, Arts et al. demonstrated that glycolysis, glutaminolysis and cholesterol synthesis are all essential for the induction of innate immune training (Arts et al., 2016). They showed that $\beta$-glucan stimulation led to enhanced glycolysis and the accumulation of TCA cycle intermediate metabolites including fumarate and succinate and these metabolites mediated epigenetic reprogramming in the form of histone modifications to train these monocytes. Glutaminolysis replenishment of the TCA cycle led to fumarate accumulation which inhibited histone demethylases, allowing methylation of histones. Arts et al. additionally showed that a similar, epigenetically-mediated induction of innate immune training was generated in response to BCG (Arts et al., 2018). A metabolite of the cholesterol synthesis pathway, mevalonate, has been shown to enhance activation of insulin-like growth factor 1 receptor (IGF1R) and activate mTOR to induce histone modifications which induce innate immune training (Bekkering et al., 2018) and that this cholesterol metabolite-mediated mechanism can be inhibited by statins. Innate immune training may have a role to play in immunity to Mtb infection. It has been well documented that there are a group of individuals termed "early clearers" who come into contact with Mtb but don't develop active infection and remain tuberculin skin test (TST) negative, indicating that they clear Mtb without inducting an adaptive immune response (Pai et al., 2016). Kaufmann et al. have demonstrated that BCG vaccination can train hematopoietic stem cells and enhance myelopoiesis, generating macrophages that are trained and can thus respond more robustly to Mtb infection in a murine model (Kaufmann et al., 2018). Furthermore, Moorlag et al. have published exciting new findings which show that $\beta$ glucan-trained macrophages are protective against Mtb infection both in a human ex vivo model and a murine in vivo model. These findings indicate that innate immune training, mediated by BCG or other trainers which induce metabolites that drive epigenetic modification, could aid in the early clearance of Mtb.

\section{MICROBIOME-DERIVED METABOLITES}

In addition to host-derived metabolites, the host microbiome is an additional source of metabolites that may influence both the survival of Mtb directly, and the host immune response. At present, the contribution of the host microbiome to Mtb disease is not well characterized, however observations from patient cohorts and studies in mouse and non-human primate models are starting to shape our understanding. HIV-infected individuals, even those on anti-retroviral treatment, are more susceptible to Mtb infection. One contributing factor may be the difference in the microbial metabolites present in the HIVlung. HIV patients have been shown to have an altered lung microbiome (Twigg et al., 2016) and Segal et al. found that short chain fatty acids (SCFA) including acetate, propionate and butyrate from lower airway anaerobic bacteria were enhanced in $\mathrm{HIV}+$ individuals and that these metabolites caused inhibition of IFN- $\gamma$ and IL17A and increased T regulatory cell generation in PBMCs from these patients stimulated ex vivo (Segal et al., 2017). Another Mtb risk factor which may be linked at least in part to microbiome metabolites is type 2 diabetes. People with type 
2 diabetes have been shown to have an altered gut microbiome (Larsen et al., 2010). An in vitro study on PBMCs showed that the SCFA butyrate suppressed pro-inflammatory cytokine expression while increasing production of IL-10 (Lachmandas et al., 2016), hinting that perhaps microbial metabolites could be playing a role in increasing Mtb susceptibility. Indole-3propionic acid (IPA), a metabolite produced by members of the gut microbiome (Wikoff et al., 2009) was shown to inhibit $\mathrm{Mtb}$ growth in vitro and to lower splenic Mtb burden in a murine model (Negatu et al., 2018). IPA is a close analog of tryptophan and was shown to exert its antimicrobial activity by acting as an inhibitor of an enzyme in the Mtb tryptophan biosynthesis pathway (Negatu et al., 2019). Our understanding of the interaction between the microbiome and its metabolites and the host immune response to Mtb is in its infancy, however these findings indicate that in addition to host-derived metabolites, microbiome-derived metabolites may have a role to play in Mtb disease.

\section{CONCLUSION}

Metabolism has emerged as a new frontier in the field of immunology, providing a better insight into the processes governing immune cell responses and providing a wealth of new therapeutic targets. Mtb infection remains a global health issue and understanding the host immune response to this bacterium is of key importance for developing novel host-directed therapies. Dysregulated metabolism is a common signature in a range of disease states beyond infection including cancer, thus therapies targeting cellular metabolic functions will have applications far beyond the scope of Mtb infection.

The burgeoning field of immunometabolism is providing exciting insights into the molecular mechanisms which govern a plethora of immune processes. Metabolites which are generated or indeed depleted by the metabolic pathways altered in response to infection are becoming appreciated as essential immune molecules rather than by-products of other processes, acting as signaling molecules, direct antimicrobial agents or conversely as fuel for the invading pathogen. Understanding how these metabolites can be harnessed to enhance Mtb treatments is of great importance. The metabolites which have been proposed to play a role in Mtb infection are summarized in Table $\mathbf{1 .}$ Dietary and pharmacological interventions which can alter the metabolites present at the site of infection may have potential to work in tandem with current treatments and vaccination programs to generate a more effective environment in which our immune systems can tackle Mtb. Repurposing existing drugs as supplemental agents in tandem with existing tuberculosis treatments may hold particular promise. For example, metformin, a drug commonly prescribed to type 2 diabetics, is an insulin sensitizer which targets complex I of the electron transport chain (El-Mir et al., 2000; Owen et al., 2000) and has been proposed as a supplementary therapy for Mtb which targets host metabolism (Oglesby et al., 2019). Epidemiological evidence has indicated that metformin both lowers the risk of developing active tuberculosis and lowers
TABLE 1 | Metabolites implicated in Mtb infection.

\begin{tabular}{|c|c|c|}
\hline \multirow[t]{2}{*}{ Metabolite } & \multicolumn{2}{|c|}{ Proposed Role in Mtb Infection } \\
\hline & Host Protection & Mtb Persistence \\
\hline Lactate & Directly anti-microbial & Fuel source for Mtb \\
\hline \multirow[t]{3}{*}{ Succinate } & HIF-1 $\alpha$ stabilization & \\
\hline & $\begin{array}{l}\text { Mitochondrial ROS } \\
\text { generation }\end{array}$ & \\
\hline & $\begin{array}{l}\text { Enhanced production of } \\
\text { IL-1 } 1 \beta \text { through SUCNR1 } \\
\text { signaling }\end{array}$ & \\
\hline \multirow[t]{3}{*}{ Itaconate } & $\begin{array}{l}\text { Inhibition of succinate } \\
\text { oxidation }\end{array}$ & \\
\hline & $\begin{array}{l}\text { Limiting of inflammatory } \\
\text { gene expression }\end{array}$ & \\
\hline & $\begin{array}{l}\text { Inhibition of the Mtb } \\
\text { enzyme ICL }\end{array}$ & \\
\hline \multirow[t]{2}{*}{ Cholesterol } & & $\begin{array}{l}\text { Inhibition of phagosomal } \\
\text { maturation and autophagy }\end{array}$ \\
\hline & & Fuel source for Mtb \\
\hline \multirow{2}{*}{$\begin{array}{l}\text { Oxidized low density } \\
\text { lipoprotein (oxLDL) }\end{array}$} & & Cholesterol accumulation \\
\hline & & $\begin{array}{l}\text { Inhibition of lysosome } \\
\text { function }\end{array}$ \\
\hline \multirow[t]{2}{*}{ Fatty acids } & & Fuel source for Mtb \\
\hline & & $\begin{array}{l}\text { Inhibition of autophagy } \\
\text { and lysosomal } \\
\text { acidification }\end{array}$ \\
\hline Kynurenine & & $\begin{array}{l}\text { Immunosuppression } \\
\text { which allows Mtb growth }\end{array}$ \\
\hline $\begin{array}{l}\text { Arginine polyamine } \\
\text { metabolites }\end{array}$ & & Inhibition of iNOS \\
\hline Citrulline & $\begin{array}{l}\text { Replaces depleted } \\
\text { arginine for the production } \\
\text { of NO by iNOS }\end{array}$ & \\
\hline Glutamine & $\begin{array}{l}\text { Fuels anti-Mtb } \\
\text { macrophage responses } \\
\text { including IL-1 } \beta \text { production } \\
\text { and NO generation }\end{array}$ & \\
\hline
\end{tabular}

the associated mortality rate (Tseng, 2018; Zhang and He, 2020) while it has been shown to improve mycobacterial containment and reduce pulmonary pathology in a murine model of Mtb infection (Singhal et al., 2014), acting through multiple mechanisms centered around cellular metabolism including mitochondrial ROS generation (Lachmandas et al., 2019). Many questions still remain to be answered, the precise role of the metabolites discussed here and their mechanisms of action are not well defined. Moving forward, comprehensive carbon tracing experiments over a time course of infection with both live and dead Mtb may elucidate the kinetics of metabolism during infection and reveal the metabolites which are being actively altered by virulent $\mathrm{Mtb}$ to aid its persistence. Additionally, specific metabolites and metabolic processes which have been identified as having immunometabolic roles in other contexts should be explored in relation to Mtb infection, for example the role of flux through the PPP and the 
relevance of the accumulation of TCA intermediates including succinate and itaconate in the case of $\mathrm{Mtb}$ infection are basic questions which have yet to be properly addressed. Thus, if we want to use host-directed therapies to win the war against Mtb, we must ensure our army of immune processes is well fed.

\section{AUTHOR CONTRIBUTIONS}

EH conceptualized and wrote the manuscript. FS wrote and edited the manuscript. All authors contributed to the article and approved the submitted version.

\section{REFERENCES}

Abbott, D. A., Suir, E., Duong, G. H., de Hulster, E., Pronk, J. T., and van Maris, A. J. (2009). Catalase overexpression reduces lactic acid-induced oxidative stress in Saccharomyces cerevisiae. Appl. Environ. Microbiol. 75, 2320-2325. doi: 10.1128/AEM.00009-09

Adu-Gyamfi, C. G., Snyman, T., Hoffmann, C. J., Martinson, N. A., Chaisson, R. E., George, J. A., et al. (2017). Plasma indoleamine 2, 3-dioxygenase, a biomarker for tuberculosis in human immunodeficiency virus-infected Patients. Clin. Infect. Dis. 65, 1356-1358. doi: 10.1093/cid/cix550

Almeida, P. E., Silva, A. R., Maya-Monteiro, C. M., Torocsik, D., D’Avila, H., Dezso, B., et al. (2009). Mycobacterium bovis bacillus Calmette-Guerin infection induces TLR2-dependent peroxisome proliferator-activated receptor gamma expression and activation: functions in inflammation, lipid metabolism, and pathogenesis. J. Immunol. 183, 1337-1345. doi: 10.4049/jimmunol.09 00365

Arts, R. J., Novakovic, B., Ter Horst, R., Carvalho, A., Bekkering, S., Lachmandas, E., et al. (2016). Glutaminolysis and fumarate accumulation integrate immunometabolic and epigenetic programs in trained immunity. Cell Metab. 24, 807-819. doi: 10.1016/j.cmet.2016.10.008

Arts, R. J. W., Moorlag, S., Novakovic, B., Li, Y., Wang, S. Y., Oosting, M., et al. (2018). BCG vaccination protects against experimental viral infection in humans through the induction of cytokines associated with trained immunity. Cell Host Microbe 23, 89-100. doi: 10.1016/j.chom.2017.12.010

Baena, A., Cabarcas, F., Alvarez-Eraso, K. L. F., Isaza, J. P., Alzate, J. F., and Barrera, L. F. (2019). Differential determinants of virulence in two Mycobacterium tuberculosis Colombian clinical isolates of the LAM09 family. Virulence 10, 695-710. doi: 10.1080/21505594.2019.1642045

Bazzan, E., Turato, G., Tine, M., Radu, C. M., Balestro, E., Rigobello, C., et al. (2017). Dual polarization of human alveolar macrophages progressively increases with smoking and COPD severity. Respir. Res 18, 40. doi: 10.1186/s12931-017-0522-0

Beatty, W. L., Belanger, T. A., Desai, A. A., Morrison, R. P., and Byrne, G. I. (1994). Tryptophan depletion as a mechanism of gamma interferonmediated chlamydial persistence. Infect. Immun. 62, 3705-3711. doi: 10.1128/IAI.62.9.3705-3711.1994

Behar, S. M., Martin, C. J., Booty, M. G., Nishimura, T., Zhao, X., Gan, H. X., et al. (2011). Apoptosis is an innate defense function of macrophages against Mycobacterium tuberculosis. Mucosal Immunol 4, 279-287. doi: 10.1038/mi.2011.3

Behrouzi, A., Alimohammadi, M., Nafari, A. H., Yousefi, M. H., Riazi Rad, F., Vaziri, F., et al. (2019). The role of host miRNAs on Mycobacterium tuberculosis. ExRNA 1:40. doi: 10.1186/s41544-019-0040-y

Bekkering, S., Arts, R. J. W., Novakovic, B., Kourtzelis, I. C., van der Heijden, L. Y., Popa, C. D., et al. (2018). Metabolic induction of trained immunity through the mevalonate pathway. Cell 172, 135-146. doi: 10.1016/j.cell.2017.11.025

Belladonna, M. L., Puccetti, P., Orabona, C., Fallarino, F., Vacca, C., Volpi, C., et al. (2007). Immunosuppression via tryptophan catabolism: the role of kynurenine pathway enzymes. Transplantation 84(1 Suppl): S17-20. doi: 10.1097/01.tp.0000269199.16209.22

\section{FUNDING}

Support for this work came from Enterprise Ireland Innovation Partnership IP/2018/0710 and Science Foundation Ireland Starting Investigators Research Grant SFI/SIRG/2136 to FS.

\section{ACKNOWLEDGMENTS}

The authors would like to thank Sarah Case in the Macrophage Homeostasis group in Trinity College Dublin for proof-reading the manuscript, and the support of our funding bodies Enterprise Ireland and Science Foundation Ireland.

Billig, S., Schneefeld, M., Huber, C., Grassl, G. A., Eisenreich, W., and Bange, F. C. (2017). Lactate oxidation facilitates growth of Mycobacterium tuberculosis in human macrophages. Sci. Rep. 7:6484. doi: 10.1038/s41598-017-05916-7

Biswas, S. K., Chittezhath, M., Shalova, I. N., and Lim, J. Y. (2012). Macrophage polarization and plasticity in health and disease. Immunol. Res. 53, 11-24. doi: 10.1007/s12026-012-8291-9

Braverman, J., Sogi, K. M., Benjamin, D., Nomura, D. K., and Stanley, S. A. (2016). HIF-1alpha is an essential Mediator of IFN-gamma-dependent immunity to mycobacterium tuberculosis. J. Immunol. 197, 1287-1297. doi: 10.4049/jimmunol.1600266

Braverman, J., and Stanley, S. A. (2017). Nitric oxide modulates macrophage responses to mycobacterium tuberculosis infection through activation of HIF-1alpha and repression of NF-kappaB. J. Immunol 199, 1805-1816. doi: 10.4049/jimmunol.1700515

Brown, A. J., Mander, E. L., Gelissen, I. C., Kritharides, L., Dean, R. T., and Jessup, W. (2000). Cholesterol and oxysterol metabolism and subcellular distribution in macrophage foam cells. Accumulation of oxidized esters in lysosomes. J. Lipid Res. 41, 226-237.

Brynildsrud, O. B., Pepperell, C. S., Suffys, P., Grandjean, L., Monteserin, J., Debech, N., et al. (2018). Global expansion of Mycobacterium tuberculosis lineage 4 shaped by colonial migration and local adaptation. Sci. Adv. 4:eaat5869. doi: 10.1126/sciadv.aat5869

Byers, D. E., and Holtzman, M. J. (2011). Alternatively activated macrophages and airway disease. Chest 140, 768-774. doi: 10.1378/chest.10-2132

Byrne, A. J., Powell, J. E., O’Sullivan, B. J., Ogger, P. P., Hoffland, A., Cook, J., et al. (2020). Dynamics of human monocytes and airway macrophages during healthy aging and after transplant. J. Exp. Med 217(3). doi: $10.1084 /$ jem.20191236

Byrne, G. I., Lehmann, L. K., and Landry, G. J. (1986). Induction of tryptophan catabolism is the mechanism for gamma-interferon-mediated inhibition of intracellular Chlamydia psittaci replication in T24 cells. Infect. Immun. 53, 347-351. doi: 10.1128/IAI.53.2.347-351.1986

Chandra, P., and Kumar, D. (2016). Selective autophagy gets more selective: Uncoupling of autophagy flux and xenophagy flux in Mycobacterium tuberculosis-infected macrophages. Autophagy 12, 608-609. doi: 10.1080/15548627.2016.1139263

Cheng, S. C., Quintin, J., Cramer, R. A., Shepardson, K. M., Saeed, S., Kumar, V., et al. (2014). mTOR- and HIF-1alpha-mediated aerobic glycolysis as metabolic basis for trained immunity. Science 345:1250684. doi: 10.1126/science.1250684

Christofk, H. R., Vander Heiden, M. G., Harris, M. H., Ramanathan, A., Gerszten, R. E., Wei, R., et al. (2008). The M2 splice isoform of pyruvate kinase is important for cancer metabolism and tumor growth. Nature 452, 230-233. doi: 10.1038/nature06734

Cohen, S. B., Gern, B. H., Delahaye, J. L., Adams, K. N., Plumlee, C. R., Winkler, J. K., et al. (2018). Alveolar Macrophages Provide an Early Mycobacterium tuberculosis Niche and Initiate Dissemination. Cell Host Microbe 24, 439-446. doi: 10.1016/j.chom.2018.08.001

Colegio, O. R., Chu, N. Q., Szabo, A. L., Chu, T., Rhebergen, A. M., Jairam, V., et al. (2014). Functional polarization of tumor-associated macrophages by tumor-derived lactic acid. Nature 513, 559-563. doi: 10.1038/nature13490 
Cumming, B. M., Addicott, K. W., Adamson, J. H., and Steyn, A. J. (2018). Mycobacterium tuberculosis induces decelerated bioenergetic metabolism in human macrophages. Elife 7:e39169. doi: 10.7554/eLife.39169.018

Davies, L. C., Jenkins, S. J., Allen, J. E., and Taylor, P. R. (2013). Tissue-resident macrophages. Nat. Immunol. 14, 986-995. doi: 10.1038/ni.2705

D’Avila, H., Melo, R. C., Parreira, G. G., Werneck-Barroso, E. H. C., CastroFaria-Neto and Bozza, P. T. (2006). Mycobacterium bovis bacillus CalmetteGuerin induces TLR2-mediated formation of lipid bodies: intracellular domains for eicosanoid synthesis in vivo. J. Immunol. 176, 3087-3097. doi: 10.4049/jimmunol.176.5.3087

Dkhar, H. K., Nanduri, R., Mahajan, S., Dave, S., Saini, A., Somavarapu, A. K., et al. (2014). Mycobacterium tuberculosis keto-mycolic acid and macrophage nuclear receptor TR4 modulate foamy biogenesis in granulomas: a case of a heterologous and noncanonical ligand-receptor pair. J. Immunol. 193, 295-305. doi: 10.4049/jimmunol.1400092

Dockrell, H. M., and Smith, S. G. (2017). What have we learnt about BCG vaccination in the last 20 years? Front. Immunol 8:1134. doi: $10.3389 /$ fimmu.2017.01134

Dodd, C. E., Pyle, C. J., Glowinski, R., Rajaram, M. V., and Schlesinger, L. S. (2016). CD36-mediated uptake of surfactant lipids by human macrophages promotes intracellular growth of mycobacterium tuberculosis. J. Immunol. 197, 4727-4735. doi: 10.4049/jimmunol.1600856

El Kasmi, K. C., Qualls, J. E., Pesce, J. T., Smith, A. M., Thompson, R. W., HenaoTamayo, M., et al. (2008). Toll-like receptor-induced arginase 1 in macrophages thwarts effective immunity against intracellular pathogens. Nat. Immunol. 9, 1399-1406. doi: 10.1038/ni.1671

El-Mir, M. Y., Nogueira, V., Fontaine, E., Averet, N., Rigoulet, M., and Leverve, X. (2000). Dimethylbiguanide inhibits cell respiration via an indirect effect targeted on the respiratory chain complex I. J. Biol. Chem. 275, 223-228. doi: $10.1074 / j b c .275 .1 .223$

Epelman, S., Lavine, K. J., Beaudin, A. E., Sojka, D. K., Carrero, J. A., Calderon, B., et al. (2014). Embryonic and adult-derived resident cardiac macrophages are maintained through distinct mechanisms at steady state and during inflammation. Immunity 40, 91-104. doi: 10.1016/j.immuni.2013.11.019

Errea, A., Cayet, D., Marchetti, P., Tang, C., Kluza, J., Offermanns, S., et al. (2016). Lactate inhibits the pro-inflammatory response and metabolic reprogramming in murine macrophages in a GPR81-independent manner. PLoS ONE 11:e0163694. doi: 10.1371/journal.pone.0163694

Fang, H. Y., Hughes, R., Murdoch, C., Coffelt, S. B., Biswas, S. K., Harris, A. L., et al. (2009). Hypoxia-inducible factors 1 and 2 are important transcriptional effectors in primary macrophages experiencing hypoxia. Blood 114, 844-859. doi: 10.1182/blood-2008-12-195941

Fernandez-Garcia, M., Rey-Stolle, F., Boccard, J., Reddy, V. P., Garcia, A., Cumming, B. M., et al. (2020). Comprehensive examination of the mouse lung metabolome following mycobacterium tuberculosis infection using a multiplatform mass spectrometry approach. J. Proteome Res 19, 2053-2070. doi: 10.1021 /acs.jproteome.9b00868

Ferreira, C., Mesquita, I., Barbosa, A. M., Osorio, N. S., Torrado, E., Beauparlant, C. J., et al. (2020). Glutamine supplementation improves the efficacy of miltefosine treatment for visceral leishmaniasis. PLoS Negl. Trop. Dis 14:e0008125. doi: 10.1371/journal.pntd.0008125

Foreman, T. W., Mehra, S., LoBato, D. N., Malek, A., Alvarez, X., Golden, N. A., et al. (2016). CD4+ T-cell-independent mechanisms suppress reactivation of latent tuberculosis in a macaque model of HIV coinfection. Proc. Natl. Acad. Sci. U.S.A. 113, E5636-E5644. doi: 10.1073/pnas.1611987113

Freemerman, A. J., Johnson, A. R., Sacks, G. N., Milner, J. J., Kirk, E. L., Troester, M. A., et al. (2014). Metabolic reprogramming of macrophages: glucose transporter 1 (GLUT1)-mediated glucose metabolism drives a proinflammatory phenotype. J. Biol. Chem. 289, 7884-7896. doi: 10.1074/jbc.M113.522037

Garaude, J., Acin-Perez, R., Martinez-Cano, S., Enamorado, M., Ugolini, M., Nistal-Villan, E., et al. (2016). Mitochondrial respiratory-chain adaptations in macrophages contribute to antibacterial host defense. Nat. Immunol. 17, 1037-1045. doi: 10.1038/ni.3509

Gautam, U. S., Foreman, T. W., Bucsan, A. N., Veatch, A. V., Alvarez, X., Adekambi, T., et al. (2018). In vivo inhibition of tryptophan catabolism reorganizes the tuberculoma and augments immune-mediated control of Mycobacterium tuberculosis. Proc. Natl. Acad. Sci. U.S.A. 115, E62-E71. doi: $10.1073 /$ pnas. 1711373114
Gautier, E. L., Shay, T., Miller, J., Greter, M., Jakubzick, C., Ivanov, S., et al. (2012). Gene-expression profiles and transcriptional regulatory pathways that underlie the identity and diversity of mouse tissue macrophages. Nat. Immunol. 13, 1118-1128. doi: 10.1038/ni.2419

Ginhoux, F., Greter, M., Leboeuf, M., Nandi, S., See, P., Gokhan, S., et al. (2010). Fate mapping analysis reveals that adult microglia derive from primitive macrophages. Science 330, 841-845. doi: 10.1126/science.1194637

Gleeson, L. E., O'Leary, S. M., Ryan, D., McLaughlin, A. M., Sheedy, F. J., and Keane, J. (2018). Cigarette Smoking Impairs the Bioenergetic Immune Response to Mycobacterium tuberculosis Infection. Am. J. Respir. Cell Mol. Biol. 59, 572-579. doi: 10.1165/rcmb.2018-0162OC

Gleeson, L. E., Sheedy, F. J. E. M., Palsson-McDermott, T, D., O’Leary, S. M., O'Sullivan, M. P., O'Neill, L. A., et al. (2016). Cutting edge: mycobacterium tuberculosis induces aerobic glycolysis in human alveolar macrophages that is required for control of intracellular bacillary replication. J. Immunol. 196, 2444-2449. doi: 10.4049/jimmunol.1501612

Gottlieb, E. (2011). p53 guards the metabolic pathway less travelled. Nat. Cell Biol. 13, 195-197. doi: $10.1038 /$ ncb2177

Graham, J. E., and Clark-Curtiss, J. E. (1999). Identification of Mycobacterium tuberculosis RNAs synthesized in response to phagocytosis by human macrophages by selective capture of transcribed sequences (SCOTS). Proc. Natl. Acad. Sci. U.S.A. 96, 11554-11559. doi: 10.1073/pnas.96.20.11554

Guerrini, V., Prideaux, B., Blanc, L., Bruiners, N., Arrigucci, R., Singh, S., et al. (2018). Storage lipid studies in tuberculosis reveal that foam cell biogenesis is disease-specific. PLoS Pathog 14:e1007223. doi: 10.1371/journal.ppat.10 07223

Guilliams, M., De Kleer, I., Henri, S., Post, S., Vanhoutte, L., De Prijck, S., et al. (2013). Alveolar macrophages develop from fetal monocytes that differentiate into long-lived cells in the first week of life via GM-CSF. J. Exp. Med. 210, 1977-1992. doi: 10.1084/jem.20131199

Guirado, E., Rajaram, M. V., Chawla, A., Daigle, J., La Perle, K. M., Arnett, E., et al. (2018). Deletion of PPARgamma in lung macrophages provides an immunoprotective response against $\mathrm{M}$. tuberculosis infection in mice. Tuberculosis (Edinb) 111, 170-177. doi: 10.1016/j.tube.2018.06.012

Hackett, E. E., Charles-Messance, H., O'Leary, S. M., Gleeson, L. E., Munoz-Wolf, N., Case, S., et al. (2020). Mycobacterium tuberculosis limits host glycolysis and IL-1beta by restriction of PFK-M via MicroRNA-21. Cell Rep. 30, 124-136. doi: 10.1016/j.celrep.2019.12.015

Hard, G. C. (1970). Some biochemical aspects of the immune macrophage. Br. J. Exp. Pathol. 51, 97-105.

Haschemi, A., Kosma, P., Gille, L., Evans, C. R., Burant, C. F., Starkl, P., et al. (2012). The sedoheptulose kinase CARKL directs macrophage polarization through control of glucose metabolism. Cell Metab. 15, 813-826. doi: 10.1016/j.cmet.2012.04.023

He, L., Weber, K. J., and Schilling, J. D. (2016). Glutamine modulates macrophage lipotoxicity. Nutrients 8:215. doi: 10.3390/nu8040215

He, W., Miao, F. J., Lin, D. C., Schwandner, R. T., Wang, Z., Gao, J., et al. (2004). Citric acid cycle intermediates as ligands for orphan G-protein-coupled receptors. Nature 429, 188-193. doi: 10.1038/nature02488

Howard, N. C., Marin, N. D., Ahmed, M., Rosa, B. A., Martin, J., Bambouskova, M., et al. (2018). Mycobacterium tuberculosis carrying a rifampicin drug resistance mutation reprograms macrophage metabolism through cell wall lipid changes. Nat. Microbiol. 3, 1099-1108. doi: 10.1038/s41564-018-0245-0

Hu, S., He, W., Du, X., Huang, Y., Fu, Y., Yang, Y., et al. (2018). Vitamin B1 helps to limit mycobacterium tuberculosis growth via regulating innate immunity in a peroxisome proliferator-activated receptor-gamma-dependent manner. Front. Immunol 9:1778. doi: 10.3389/fimmu.2018.01778

Huang, L., Nazarova, E. V., Tan, S., Liu, Y., and Russell, D. G. (2018), Growth of Mycobacterium tuberculosis in vivo segregates with host macrophage metabolism and ontogeny. J. Exp. Med. 215, 1135-1152. doi: $10.1084 /$ jem. 20172020

Huang, Z., Luo, Q., Guo, Y., Chen, J., Xiong, G., Peng, Y., et al. (2015). Mycobacterium tuberculosis-induced polarization of human macrophage orchestrates the formation and development of tuberculous granulomas in vitro. PLoS ONE 10:e0129744. doi: 10.1371/journal.pone.0129744

Huynh, K. K., Gershenzon, E., and Grinstein, S. (2008). Cholesterol accumulation by macrophages impairs phagosome maturation. J. Biol. Chem. 283, 35745-35755. doi: 10.1074/jbc.M806232200 
Infantino, V., Convertini, P., Cucci, L., Panaro, M. A., Di Noia, M. A., Calvello, R., et al. (2011). The mitochondrial citrate carrier: a new player in inflammation. Biochem. J. 438, 433-436. doi: 10.1042/BJ20111275

Jha, A. K., Huang, S. C., Sergushichev, A., Lampropoulou, V., Ivanova, Y., Loginicheva, E., et al. (2015). Network integration of parallel metabolic and transcriptional data reveals metabolic modules that regulate macrophage polarization. Immunity 42, 419-430. doi: 10.1016/j.immuni.2015.02.005

Kang, D. D., Lin, Y., Moreno, J. R., Randall, T. D., and Khader, S. A. (2011). Profiling early lung immune responses in the mouse model of tuberculosis. PLoS ONE 6:e16161. doi: 10.1371/journal.pone.0016161

Kaufmann, E., Sanz, J., Dunn, J. L., Khan, N., Mendonca, L. E., Pacis, A., et al. (2018). BCG Educates hematopoietic stem cells to generate protective innate immunity against tuberculosis. Cell 172, 176-190. doi: 10.1016/j.cell.2017.12.031

Keiran, N., Ceperuelo-Mallafre, V., Calvo, E., Hernandez-Alvarez, M. I., Ejarque, M., Nunez-Roa, C., et al. (2019). SUCNR1 controls an anti-inflammatory program in macrophages to regulate the metabolic response to obesity. Nat. Immunol. 20, 581-592. doi: 10.1038/s41590-019-0372-7

Kelly, B., and O’Neill, L. A. (2015). Metabolic reprogramming in macrophages and dendritic cells in innate immunity. Cell Res. 25, 771-784. doi: $10.1038 / \mathrm{cr} .2015 .68$

Kelly, D. M., ten Bokum, A. M., O'Leary, S. M., O’Sullivan, M. P., and Keane, J. (2008). Bystander macrophage apoptosis after Mycobacterium tuberculosis H37Ra infection. Infect. Immun 76, 351-360. doi: 10.1128/IAI.00614-07

Kim, M. J., Wainwright, H. C., Locketz, M., Bekker, L. G., Walther, G. B., Dittrich, C., et al. (2010). Caseation of human tuberculosis granulomas correlates with elevated host lipid metabolism. EMBO Mol. Med. 2, 258-274. doi: 10.1002/emmm.201000079

Kleinnijenhuis, J., Quintin, J., Preijers, F., Joosten, L. A., Ifrim, D. C., Saeed, S., et al. (2012). Bacille Calmette-Guerin induces NOD2-dependent nonspecific protection from reinfection via epigenetic reprogramming of monocytes. Proc. Natl. Acad. Sci. U.S.A. 109, 17537-17542. doi: 10.1073/pnas.1202870109

Knight, M., Braverman, J., Asfaha, K., Gronert, K., and Stanley, S. (2018). Lipid droplet formation in Mycobacterium tuberculosis infected macrophages requires IFN-gamma/HIF-1alpha signaling and supports host defense. PLoS Pathog 14:e1006874. doi: 10.1371/journal.ppat.1006874

Koeken, V., Lachmandas, E., Riza, A., Matzaraki, V., Li, Y., Kumar, V., et al. (2019). Role of Glutamine metabolism in host defense against mycobacterium tuberculosis infection. J. Infect. Dis. 219, 1662-1670. doi: 10.1093/infdis/j iy709

Lachmandas, E., Eckold, C., Bohme, J., Koeken, V., Marzuki, M. B., Blok, B., et al. (2019). Metformin alters human host responses to mycobacterium tuberculosis in healthy subjects. J. Infect. Dis. 220, 139-150. doi: 10.1093/infdis/jiz064

Lachmandas, E. C. N., van den Heuvel, Damen, M. S., Cleophas, M. C., Netea, M. G., and van Crevel, R. (2016). Diabetes mellitus and increased tuberculosis susceptibility: the role of short-chain fatty acids. J. Diabetes Res. 2016:6014631. doi: $10.1155 / 2016 / 6014631$

Lampropoulou, V., Sergushichev, A., Bambouskova, M., Nair, S., Vincent, E. E., Loginicheva, E., et al. (2016). Itaconate links inhibition of succinate dehydrogenase with macrophage metabolic remodeling and regulation of inflammation. Cell Metab. 24, 158-166. doi: 10.1016/j.cmet.2016.06.004

Lange, S. M., McKell, M. C., Schmidt, S. M., Hossfeld, A. P., Chaturvedi, V., Kinder, J. M., et al. (2017). 1-Citrulline metabolism in mice augments $\mathrm{CD} 4(+) \mathrm{T}$ cell proliferation and cytokine production in vitro, and accumulation in the mycobacteria-infected lung. Front. Immunol 8:1561. doi: 10.3389 /fimmu.2017.01561

Lange, S. M., McKell, M. C., Schmidt, S. M., Zhao, J., Crowther, R. R., Green, L. C., et al. (2019). l-arginine synthesis from l-citrulline in myeloid cells drives host defense against mycobacteria in vivo. J. Immunol. 202, 1747-1754. doi: 10.4049/jimmunol.1801569

Larsen, N., Vogensen, F. K. F. W., van den Berg, N.ielsen, D. S., Andreasen, A. S., Pedersen, B. K., Al-Soud, W. A., et al. (2010). Gut microbiota in human adults with type 2 diabetes differs from non-diabetic adults. PLOS ONE 5:e9085. doi: 10.1371/journal.pone.0009085

Lee, W., VanderVen, B. C., Fahey, R. J., and Russell, D. G. (2013). Intracellular Mycobacterium tuberculosis exploits host-derived fatty acids to limit metabolic stress. J. Biol. Chem. 288, 6788-6800. doi: 10.1074/jbc.M112.445056
Li, Z., Xu, G., Qin, Y., Zhang, C., Tang, H., Yin, Y., et al. (2014). Ghrelin promotes hepatic lipogenesis by activation of mTOR-PPARgamma signaling pathway. Proc. Natl. Acad. Sci. U.S.A. 111, 13163-13168. doi: 10.1073/pnas.1411571111

Littlewood-Evans, A., Sarret, S., Apfel, V., Loesle, P., Dawson, J., Zhang, J., et al. (2016). GPR91 senses extracellular succinate released from inflammatory macrophages and exacerbates rheumatoid arthritis. J. Exp. Med. 213, 1655-1662. doi: 10.1084/jem.20160061

Lorenz, M. C., and Fink, G. R. (2002). Life and death in a macrophage: role of the glyoxylate cycle in virulence. Eukaryotic Cell 1, 657-662. doi: 10.1128/EC.1.5.657-662.2002

Luan, H. H., and Medzhitov, R. (2016). Food fight: role of itaconate and other metabolites in antimicrobial defense. Cell Metab 24, 379-387. doi: 10.1016/j.cmet.2016.08.013

Manyazewal, T., Woldeamanuel, Y., Blumberg, H. M., Fekadu, A., and Marconi, V. C. (2020). The fight to end tuberculosis must not be forgotten in the COVID-19 outbreak. Nat Med. 26, 811-812. doi: 10.1038/s41591-020-0917-1

Marrero, J., Rhee, K. Y., Schnappinger, D., Pethe, K., and Ehrt, S. (2010). Gluconeogenic carbon flow of tricarboxylic acid cycle intermediates is critical for Mycobacterium tuberculosis to establish and maintain infection. Proc. Natl. Acad. Sci. U.S.A. 107, 9819-9824. doi: 10.1073/pnas.1000715107

Martens, G. W., Arikan, M. C., Lee, J., Ren, F., Vallerskog, T., and Kornfeld, H. (2008). Hypercholesterolemia impairs immunity to tuberculosis. Infect. Immun 76, 3464-3472. doi: 10.1128/IAI.00037-08

Martin, C. J., Booty, M. G., Rosebrock, T. R., Nunes-Alves, C., Desjardins, D. M., Keren, I., et al. (2012). Efferocytosis is an innate antibacterial mechanism. Cell Host Microbe 12, 289-300. doi: 10.1016/j.chom.2012.06.010

Mattila, J. T., Ojo, O. O., Kepka-Lenhart, D., Marino, S., Kim, J. H., Eum, S. Y., et al. (2013). Microenvironments in tuberculous granulomas are delineated by distinct populations of macrophage subsets and expression of nitric oxide synthase and arginase isoforms. J. Immunol. 191, 773-784. doi: 10.4049/jimmunol.1300113

Maus, U. A., Janzen, S., Wall, G., Srivastava, M., Blackwell, T. S., Christman, J. W., et al. (2006). Resident alveolar macrophages are replaced by recruited monocytes in response to endotoxin-induced lung inflammation. Am. J. Respir. Cell Mol. Biol. 35, 227-235. doi: 10.1165/rcmb.2005-0241OC

Mayer-Barber, K. D., and Sher, A. (2015). Cytokine and lipid mediator networks in tuberculosis. Immunol. Rev. 264, 264-275. doi: 10.1111/imr.12249

McKinney, J. D. K., Honer zu Bentrup, Munoz-Elias, E. J., Miczak, A., Chen, B., Chan, W. T., Swenson, D., et al. (2000). Persistence of Mycobacterium tuberculosis in macrophages and mice requires the glyoxylate shunt enzyme isocitrate lyase. Nature 406, 735-738. doi: 10.1038/35021074

Mehra, S., Alvarez, X., Didier, P. J., Doyle, L. A., Blanchard, J. L., Lackner, A. A., et al. (2013). Granuloma correlates of protection against tuberculosis and mechanisms of immune modulation by Mycobacterium tuberculosis. J. Infect. Dis. 207, 1115-1127. doi: 10.1093/infdis/jis778

Michelucci, A., Cordes, T., Ghelfi, J., Pailot, A., Reiling, N., Goldmann, O., et al. (2013). Immune-responsive gene 1 protein links metabolism to immunity by catalyzing itaconic acid production. Proc. Natl. Acad. Sci. U.S.A. 110, 7820-7825. doi: 10.1073/pnas.1218599110

Mills, C. D., Kincaid, K., Alt, J. M., Heilman, M. J., and Hill, A. M. (2000). M1/M-2 macrophages and the Th1/Th2 paradigm. J. Immunol. 164, 6166-6173. doi: 10.4049/jimmunol.164.12.6166

Mills, E. L., Kelly, B., Logan, A., Costa, A. S. H., Varma, M., Bryant, C. E., et al. (2016). Succinate dehydrogenase supports metabolic repurposing of mitochondria to drive inflammatory macrophages. Cell 167, 457-470. doi: 10.1016/j.cell.2016.08.064

Mills, E. L., Ryan, D. G., Prag, H. A., Dikovskaya, D., Menon, D., Zaslona, Z., et al. (2018). Itaconate is an anti-inflammatory metabolite that activates Nrf2 via alkylation of KEAP1. Nature 556, 113-117. doi: 10.1038/nature25986

Momen-Heravi, F., and Bala, S. (2018). miRNA regulation of innate immunity. J. Leukoc Biol. 103, 1205-1217. doi: 10.1002/JLB.3MIR1117-459R

Moffett, J. R., and Namboodiri, M. A. (2003). Tryptophan and the immune response. Immunol. Cell Biol 81, 247-265. doi: 10.1046/j.1440-1711.2003.t01-1-01177.x

Moore, K. J., Sheedy, F. J., and Fisher, E. A. (2013). Macrophages in atherosclerosis: a dynamic balance. Nat. Rev. Immunol 13, 709-721. doi: 10.1038/n ri3520 
Munn, D. H., Zhou, M., Attwood, J. T., Bondarev, I., Conway, S. J., Marshall, B., et al. (1998). Prevention of allogeneic fetal rejection by tryptophan catabolism. Science 281, 1191-1193. doi: 10.1126/science.281.5380.1191

Munoz-Elias, E. J., and McKinney, J. D. (2005). Mycobacterium tuberculosis isocitrate lyases 1 and 2 are jointly required for in vivo growth and virulence. Nat. Med. 11, 638-644. doi: 10.1038/nm1252

Murphy, C., and Newsholme, P. (1998). Importance of glutamine metabolism in murine macrophages and human monocytes to L-arginine biosynthesis and rates of nitrite or urea production. Clin. Sci. 95, 397-407. doi: $10.1042 / \operatorname{cs} 0950397$

Murray, P. J. (2016). Amino acid auxotrophy as a system of immunological control nodes. Nat. Immunol. 17, 132-139. doi: 10.1038/ni.3323

Nair, S., Huynh, J. P., Lampropoulou, V., Loginicheva, E., Esaulova, E., Gounder, A. P., et al. (2018). Irg1 expression in myeloid cells prevents immunopathology during M. tuberculosis infection. J. Exp. Med. 215, 1035-1045. doi: 10.1084/jem.20180118

Negatu, D. A., Liu, J. J. J., Zimmerman, M., Kaya, F., Dartois, V., Aldrich, C. C., et al. (2018). Whole-cell screen of fragment library identifies gut microbiota metabolite indole propionic acid as antitubercular. Antimicrob. Agents Chemother 62:3. doi: 10.1128/AAC.01571-17

Negatu, D. A., Yamada, Y., Xi, Y., Go, M. L., Zimmerman, M., Ganapathy, U., et al. (2019). Gut microbiota metabolite indole propionic acid targets tryptophan biosynthesis in mycobacterium tuberculosis. MBio 10:2. doi: $10.1128 / \mathrm{mBio} .02781-18$

Netea, M. G., Quintin, J., and, J. W., van der Meer (2011). Trained immunity: a memory for innate host defense. Cell Host Microbe 9, 355-361. doi: 10.1016/j.chom.2011.04.006

Nourshargh, S., and Alon, R. (2014). Leukocyte migration into inflamed tissues. Immunity 41, 694-707. doi: 10.1016/j.immuni.2014.10.008

Oglesby, W., Kara, A. M., Granados, H., and Cervantes, J. L. (2019). Metformin in tuberculosis: beyond control of hyperglycemia. Infection 47, 697-702. doi: 10.1007/s15010-019-01322-5

O'Neill, L. A., Kishton, R. J., and Rathmell, J. (2016). A guide to immunometabolism for immunologists. Nat. Rev. Immunol. 16, 553-565. doi: $10.1038 /$ nri.2016.70

Osada-Oka, M., Goda, N., Saiga, H., Yamamoto, M., Takeda, K., Ozeki, Y., et al. (2019). Metabolic adaptation to glycolysis is a basic defense mechanism of macrophages for Mycobacterium tuberculosis infection. Int. Immunol. 31, 781-793. doi: 10.1093/intimm/dxz048

Ouimet, M., Koster, S., Sakowski, E., Ramkhelawon, B., van Solingen, C., Oldebeken, S., et al. (2016). Mycobacterium tuberculosis induces the miR-33 locus to reprogram autophagy and host lipid metabolism. Nat. Immunol. 17, 677-686. doi: 10.1038/ni.3434

Owen, M. R., Doran, E., and Halestrap, A. P. (2000). Evidence that metformin exerts its anti-diabetic effects through inhibition of complex 1 of the mitochondrial respiratory chain. Biochem. J. 348, 607-614. doi: 10.1042/bj3480607

Pai, M., Behr, M. A., Dowdy, D., Dheda, K., Divangahi, M., Boehme, C. C., et al. (2016). Tuberculosis. Nat. Rev. Dis. Primers 2:16076. doi: 10.1038/nrdp.2016.76

Palanisamy, G. S., Kirk, N. M., Ackart, D. F., Obregon-Henao, A., Shanley, C. A., Orme, I. M., et al. (2012). Uptake and accumulation of oxidized low-density lipoprotein during Mycobacterium tuberculosis infection in guinea pigs. PLoS ONE 7:e34148. doi: 10.1371/journal.pone.0034148

Palazon, A., Goldrath, A. W., Nizet, V., and Johnson, R. S. (2014). HIF transcription factors, inflammation, and immunity. Immunity 41, 518-528. doi: 10.1016/j.immuni.2014.09.008

Palmieri, E. M., Gonzalez-Cotto, M., Baseler, W. A., Davies, L. C., Ghesquiere, B., Maio, N., et al. (2020). Nitric oxide orchestrates metabolic rewiring in M1 macrophages by targeting aconitase 2 and pyruvate dehydrogenase. Nat. Commun. 11:698. doi: 10.1038/s41467-020-14433-7

Palmieri, E. M., Menga, A., Martin-Perez, R., Quinto, A., Riera-Domingo, C., De Tullio, G., et al. (2017). Pharmacologic or genetic targeting of glutamine synthetase skews macrophages toward an M1-like phenotype and inhibits tumor metastasis. Cell Rep. 20, 1654-1666. doi: 10.1016/j.celrep.2017.07.054

Palsson-McDermott, E. M., Curtis, A. M., Goel, G., Lauterbach, M. A., Sheedy, F. J., Gleeson, L. E. M. W., et al. (2015). Pyruvate kinase M2 regulates Hif-1alpha activity and IL-1beta induction and is a critical determinant of the warburg effect in LPS-activated macrophages. Cell Metab. 21, 65-80. doi: 10.1016/j.cmet.2014.12.005

Pandey, A. K., and Sassetti, C. M. (2008). Mycobacterial persistence requires the utilization of host cholesterol. Proc. Natl. Acad. Sci. U.S.A. 105, 4376-4380. doi: $10.1073 /$ pnas.0711159105

Pearce, E. L., and Pearce, E. J. (2013). Metabolic pathways in immune cell activation and quiescence. Immunity 38, 633-643. doi: 10.1016/j.immuni.2013.04.005

Peyron, P., Vaubourgeix, J., Poquet, Y., Levillain, F., Botanch, C., Bardou, F., et al. (2008). Foamy macrophages from tuberculous patients' granulomas constitute a nutrient-rich reservoir for M. tuberculosis persistence. PLoS Pathog 4:e1000204. doi: 10.1371/journal.ppat.1000204

Pym, A. S., Brodin, P., Brosch, R., Huerre, M., and Cole, S. T. (2002). Loss of $\mathrm{RD} 1$ contributed to the attenuation of the live tuberculosis vaccines Mycobacterium bovis BCG and Mycobacterium microti. Mol. Microbiol. 46, 709-717. doi: 10.1046/j.1365-2958.2002.03237.x

Qu, H. Q., Rentfro, A. R., Lu, Y., Nair, S., Hanis, C. L., McCormick, J. B., et al. (2012). Host susceptibility to tuberculosis: insights from a longitudinal study of gene expression in diabetes. Int. J. Tuberc. Lung Dis 16, 370-372. doi: 10.5588/ijtld.11.0536

Qualls, J. E., and Murray, P. J. (2016). Immunometabolism within the tuberculosis granuloma: amino acids, hypoxia, and cellular respiration. Semin. Immunopathol. 38, 139-152. doi: 10.1007/s00281-015-0534-0

Rajaram, M. V., Brooks, M. N., Morris, J. D., Torrelles, J. B., Azad, A. K., and Schlesinger, L. S. (2010). Mycobacterium tuberculosis activates human macrophage peroxisome proliferator-activated receptor gamma linking mannose receptor recognition to regulation of immune responses. J. Immunol. 185, 929-942. doi: 10.4049/jimmunol.1000866

Ralph, A. P., Waramori, G., Pontororing, G. J., Kenangalem, E., Wiguna, A., Tjitra, E., et al. (2013). L-arginine and vitamin D adjunctive therapies in pulmonary tuberculosis: a randomised, double-blind, placebo-controlled trial. PLoS ONE 8:e70032. doi: 10.1371/journal.pone.0070032

Rapovy, S. M., Zhao, J., Bricker, R. L., Schmidt, S. M., Setchell, K. D., and Qualls, J. E. (2015). Differential requirements for L-citrulline and L-arginine during antimycobacterial macrophage activity. J. Immunol. 195, 3293-3300. doi: 10.4049/jimmunol.1500800

Refai, A., Gritli, S., Barbouche, M. R., and Essafi, M. (2018). Mycobacterium tuberculosis virulent factor ESAT-6 drives macrophage differentiation toward the pro-inflammatory M1 phenotype and subsequently switches it to the anti-inflammatory M2 phenotype. Front. Cell. Infect. Microbiol 8:327. doi: $10.3389 /$ fcimb. 2018.00327

Rogatzki, M. J., Ferguson, B. S., Goodwin, M. L., and Gladden, L. B. (2015). Lactate is always the end product of glycolysis. Front. Neurosci 9:22. doi: 10.3389/fnins.2015.00022

Schneider, C., Nobs, S. P., Kurrer, M., Rehrauer, H., Thiele, C., and Kopf, M. (2014). Induction of the nuclear receptor PPAR-gamma by the cytokine GM-CSF is critical for the differentiation of fetal monocytes into alveolar macrophages. Nat. Immunol 15, 1026-1037. doi: 10.1038/ni.3005

Schon, T., Elias, D., Moges, F., Melese, E., Tessema, T., Stendahl, O., et al. (2003). Arginine as an adjuvant to chemotherapy improves clinical outcome in active tuberculosis. Eur. Respir. J 21, 483-488. doi: 10.1183/09031936.03.000 90702

Schulz, C., Gomez Perdiguero, E., Chorro, L., Szabo-Rogers, H., Cagnard, N., Kierdorf, K., et al. (2012). A lineage of myeloid cells independent of Myb and hematopoietic stem cells. Science 336, 86-90. doi: 10.1126/science.12 19179

Segal, L. N., Clemente, J. C., Li, Y., Ruan, C., Cao, J., Danckers, M., et al. (2017). Anaerobic bacterial fermentation products increase tuberculosis risk in antiretroviral-drug-treated HIV patients. Cell Host Microbe 21, 530-537. doi: 10.1016/j.chom.2017.03.003

Serafini, A., Tan, L., Horswell, S., Howell, S., Greenwood, D. J., Hunt, D. M., et al. (2019). Mycobacterium tuberculosis requires glyoxylate shunt and reverse methylcitrate cycle for lactate and pyruvate metabolism. Mol. Microbiol. 112, 1284-1307. doi: 10.1111/mmi.14362

Shaykhiev, R., Krause, A., Salit, J., Strulovici-Barel, Y., Harvey, B. G., O'Connor, T. P., et al. (2009). Smoking-dependent reprogramming of alveolar macrophage polarization: implication for pathogenesis of chronic obstructive pulmonary disease. J. Immunol. 183, 2867-2883. doi: 10.4049/jimmunol.0900473 
Shi, L., Jiang, Q., Bushkin, Y., Subbian, S., and Tyagi, S. (2019). Biphasic Dynamics of Macrophage Immunometabolism during Mycobacterium tuberculosis Infection. MBio 10:18. doi: 10.1128/mBio.02550-18

Shi, L., Salamon, H., Eugenin, E. A., Pine, R., Cooper, A., and Gennaro, M. L. (2015). Infection with Mycobacterium tuberculosis induces the Warburg effect in mouse lungs. Sci. Rep 5:18176. doi: 10.1038/srep18176

Shin, J. H., Yang, J. Y., Jeon, B. Y., Yoon, Y. J., Cho, S. N., Kang, Y. H., et al. (2011). (1)H NMR-based metabolomic profiling in mice infected with Mycobacterium tuberculosis. J. Proteome Res. 10, 2238-2247. doi: 10.1021/pr101054m

Singh, V., Jamwal, S., Jain, R., Verma, P., Gokhale, R., and Rao, K. V. (2012). Mycobacterium tuberculosis-driven targeted recalibration of macrophage lipid homeostasis promotes the foamy phenotype. Cell Host Microbe 12, 669-681. doi: 10.1016/j.chom.2012.09.012

Singhal, A., Jie, L., Kumar, P., Hong, G. S., Leow, M. K., Paleja, B., et al. (2014). Metformin as adjunct antituberculosis therapy. Sci. Transl. Med. 6:263ra159. doi: 10.1126/scitranslmed.3009885

Skold, M., and Behar, S. M. (2008). Tuberculosis triggers a tissue-dependent program of differentiation and acquisition of effector functions by circulating monocytes. J. Immunol. 181, 6349-6360. doi: 10.4049/jimmunol.181.9.6349

Soh, A. Z., Chee, C. B., Wang, Y. T., Yuan, J. M., and Koh, W. P. (2016). Dietary cholesterol increases the risk whereas PUFAs reduce the risk of active tuberculosis in singapore chinese. J. Nutr. 146, 1093-1100. doi: 10.3945/jn.115.228049

Somashekar, B. S., Amin, A. G., Rithner, C. D., Troudt, J., Basaraba, R., Izzo, A., et al. (2011). Metabolic profiling of lung granuloma in Mycobacterium tuberculosis infected guinea pigs: ex vivo $1 \mathrm{H}$ magic angle spinning NMR studies. J. Proteome Res. 10, 4186-4195. doi: 10.1021/pr2003352

Southan, G. J., Szabo, C., and Thiemermann, C. (1994). Inhibition of the induction of nitric oxide synthase by spermine is modulated by aldehyde dehydrogenase. Biochem. Biophys. Res. Commun. 203, 1638-1644. doi: 10.1006/bbrc.1994.2374

Strelko, C. L., Lu, W., Dufort, F. J., Seyfried, T. N., Chiles, T. C., Rabinowitz, J. D., et al. (2011). Itaconic acid is a mammalian metabolite induced during macrophage activation. J. Am. Chem. Soc 133, 16386-16389. doi: $10.1021 /$ ja2070889

Sulis, G., Centis, R., Sotgiu, G., D’Ambrosio, L., Pontali, E., Spanevello, A., et al. (2016). Recent developments in the diagnosis and management of tuberculosis. NPJ Prim. Care Respir. Med. 26:16078. doi: 10.1038/npjpcrm.2016.78

Suzuki, Y., Suda, T., Asada, K., Miwa, S., Suzuki, M., Fujie, M., et al. (2012). Serum indoleamine 2,3-dioxygenase activity predicts prognosis of pulmonary tuberculosis. Clin. Vaccine Immunol. 19, 436-442. doi: 10.1128/CVI.05 402-11

Tanimura, T., Jaramillo, E., Weil, D., Raviglione, M., and Lonnroth, K. (2014). Financial burden for tuberculosis patients in low- and middleincome countries: a systematic review. Eur. Respir. J. 43, 1763-1775. doi: 10.1183/09031936.00193413

Tannahill, G. M., Curtis, A. M., Adamik, J. E. M., Palsson-McDermott, McGettrick, A. F., Goel, G., Frezza, C., et al. (2013). Succinate is an inflammatory signal that induces IL-1beta through HIF-1alpha. Nature 496, 238-242. doi: $10.1038 /$ nature 11986

Tseng, C. H. (2018). Metformin decreases risk of tuberculosis infection in type 2 diabetes patients. J Clin Med 7:9. doi: 10.3390/jcm7090264

Twigg, H. L., 3rd, Knox, K. S., Zhou, J., Crothers, K. A., Nelson, D. E., Toh, E., et al. (2016). Effect of advanced HIV infection on the respiratory microbiome. Am. J. Respir. Crit. Care Med. 194, 226-235. doi: 10.1164/rccm.201509-1875OC

van der Heijden, C., Noz, M. P., Joosten, L. A. B., Netea, M. G., Riksen, N. P., and Keating, S. T. (2018). Epigenetics and trained immunity. Antioxid. Redox Signal 29, 1023-1040. doi: 10.1089/ars.2017.7310

Velmurugan, K., Chen, B., Miller, J. L., Azogue, S., Gurses, S., Hsu, T., et al. (2007). Mycobacterium tuberculosis nuoG is a virulence gene that inhibits apoptosis of infected host cells. PLoS Pathog 3:e110. doi: 10.1371/journal.ppat.0030110

Vrieling, F., Alisjahbana, B., Sahiratmadja, E., van Crevel, R., Harms, A. C., Hankemeier, T., et al. (2019a). Plasma metabolomics in tuberculosis patients with and without concurrent type 2 diabetes at diagnosis and during antibiotic treatment. Sci. Rep 9:18669. doi: 10.1038/s41598-019-54983-5

Vrieling, F., Wilson, L., Rensen, P. C. N., Walzl, G., Ottenhoff, T. H. M., and Joosten, S. A. (2019b). Oxidized low-density lipoprotein (oxLDL) supports Mycobacterium tuberculosis survival in macrophages by inducing lysosomal dysfunction. PLoS Pathog 15:e1007724. doi: 10.1371/journal.ppat.1007724

Wallace, C., and Keast, D. (1992). Glutamine and macrophage function. Metab. Clin. Exp. 41, 1016-1020. doi: 10.1016/0026-0495(92)90130-3

Wang, H., Fedorov, A. A., Fedorov, E. V., Hunt, D. M., Rodgers, A., Douglas, H. L., et al. (2019). An essential bifunctional enzyme in Mycobacterium tuberculosis for itaconate dissimilation and leucine catabolism. Proc. Natl. Acad. Sci. U.S.A. 116, 15907-15913. doi: 10.1073/pnas.1906606116

Weiner, J., III., Parida, S. K., Maertzdorf, J., Black, G. F., Repsilber, D., Telaar, A., et al. (2012). Biomarkers of inflammation, immunosuppression and stress with active disease are revealed by metabolomic profiling of tuberculosis patients. PLoS ONE 7:e40221. doi: 10.1371/journal.pone.0040221

Wells, S. M., Kew, S., Yaqoob, P., Wallace, F. A., and Calder, P. C. (1999). Dietary glutamine enhances cytokine production by murine macrophages. Nutrition 15, 881-884. doi: 10.1016/S0899-9007(99)00184-7

Wikoff, W. R., Anfora, A. T., Liu, J., Schultz, P. G., Lesley, S. A., Peters, E. C., et al. (2009). Metabolomics analysis reveals large effects of gut microflora on mammalian blood metabolites. Proc. Natl. Acad. Sci. U.S.A. 106, 3698-3703. doi: $10.1073 /$ pnas.0812874106

Williams, J. O., Roche, T. E., and McFadden, B. A. (1971). Mechanism of action of isocitrate lyase from Pseudomonas indigofera. Biochemistry 10, 1384-1390. doi: 10.1021/bi00784a017

Wilson, J. E. (2003). Isozymes of mammalian hexokinase: structure, subcellular localization and metabolic function. J. Exp. Biol. 206, 2049-2057. doi: $10.1242 /$ jeb.00241

World Health Organization (2019). Global Tuberculosis Report 2019. Geneva: WHO.

Wu, G. Y., and Brosnan, J. T. (1992). Macrophages can convert citrulline into arginine. Biochem J. 281, 45-48. doi: 10.1042/bj2810045

Yan, Y., Zhang, G. X., Gran, B., Fallarino, F., Yu, S., Li, H., et al. (2010). IDO upregulates regulatory $\mathrm{T}$ cells via tryptophan catabolite and suppresses encephalitogenic $\mathrm{T}$ cell responses in experimental autoimmune encephalomyelitis. J. Immunol. 185, 5953-5961. doi: 10.4049/jimmunol.1001628

Yona, S., Kim, K. W., Wolf, Y., Mildner, A., Varol, D., Breker, M., et al. (2013). Fate mapping reveals origins and dynamics of monocytes and tissue macrophages under homeostasis. Immunity 38, 79-91. doi: 10.1016/j.immuni.2012.12.001

Yurdagul, A., Jr., Subramanian, M., Wang, X., Crown, S. B., Ilkayeva, O. R., et al. (2020). Macrophage metabolism of apoptotic cell-derived arginine promotes continual efferocytosis and resolution of injury. Cell Metab. 31, 518-533. doi: 10.1016/j.cmet.2020.01.001

Zhang, M., and He, J. Q. (2020). Impacts of metformin on tuberculosis incidence and clinical outcomes in patients with diabetes: a systematic review and metaanalysis. Eur. J. Clin. Pharmacol. 76, 149-159. doi: 10.1007/s00228-019-02786-y

Zhang, Y. J., Reddy, M. C., Ioerger, T. R., Rothchild, A. C., Dartois, V., Schuster, B. M., et al. (2013). Tryptophan biosynthesis protects mycobacteria from CD4 T-cell-mediated killing. Cell 155, 1296-1308. doi: 10.1016/j.cell.2013.10.045

Conflict of Interest: The authors declare that the research was conducted in the absence of any commercial or financial relationships that could be construed as a potential conflict of interest.

Copyright (c) 2020 Hackett and Sheedy. This is an open-access article distributed under the terms of the Creative Commons Attribution License (CC BY). The use, distribution or reproduction in other forums is permitted, provided the original author(s) and the copyright owner(s) are credited and that the original publication in this journal is cited, in accordance with accepted academic practice. No use, distribution or reproduction is permitted which does not comply with these terms. 